\title{
Hilbert modular surfaces for square discriminants and elliptic subfields of genus 2 function fields
}

\author{
Abhinav Kumar ${ }^{1,2^{*}}$
}

*Correspondence:

thenav@gmail.com

2 Present Address:

Department of Mathematics,

Stony Brook University, Stony Brook, NY 11794, USA

Full list of author information

is available at the end of the article

\section{Springer}

\begin{abstract}
We compute explicit rational models for some Hilbert modular surfaces corresponding to square discriminants, by connecting them to moduli spaces of elliptic K3 surfaces. Since they parametrize decomposable principally polarized abelian surfaces, they are also moduli spaces for genus-2 curves covering elliptic curves via a map of fixed degree. We thereby extend classical work of Jacobi, Hermite, Bolza etc., and more recent work of Kuhn, Frey, Kani, Shaska, Völklein, Magaard and others, producing explicit families of reducible Jacobians. In particular, we produce a birational model for the moduli space of pairs $(C, E)$ of a genus 2 curve $C$ and elliptic curve $E$ with a map of degree $n$ from $C$ to $E$, as well as a tautological family over the base, for $2 \leq n \leq 11$. We also analyze the resulting models from the point of view of arithmetic geometry, and produce several interesting curves on them.
\end{abstract}

Keywords: K3 surfaces, Moduli spaces, Hilbert modular surfaces, Genus-2 curves, Jacobians, Elliptic curves

Mathematics Subject Classification: Primary 11F41; Secondary 14J28; $14 \mathrm{H} 40$

\section{Introduction}

In algebraic geometry and number theory, one is frequently interested in abelian varieties with extra endomorphisms, and their moduli spaces. The study of elliptic curves and their moduli spaces has been extremely influential in the last century (for instance, see $[10,22,34])$. Higher dimensional abelian varieties have also seen many applications; however, it has remained quite a challenge to provide explicit computational descriptions for these or their moduli spaces. There has been quite a lot of work in the direction of explicit approaches to abelian surfaces in the last couple of decades. This paper is chiefly concerned with moduli spaces of genus 2 curves with decomposable Jacobians. We say that an abelian variety $A$ over a field $k$ is decomposable or reducible over $k$ if it is isogenous over $k$ to a product of abelian varieties of smaller dimension.

In particular, we will be interested in principally polarized abelian surfaces $A$ which are isogenous to a product of elliptic curves. A natural way to produce such an abelian surface is as the Jacobian of a curve $C$ of genus 2, which has a map of degree $n>1$ to an elliptic curve $E$. The morphism $\phi: C \rightarrow E$ induces $\phi^{*}: E \rightarrow J(C)=A$ and $\phi_{*}: A=J(C) \rightarrow E$, with $\phi_{*} \circ \phi^{*}=n_{E}$. The curve $C$ also has a map of degree $n$ to the

(c) 2015 Kumar. This article is distributed under the terms of the Creative Commons Attribution 4.0 International License (http:// creativecommons.org/licenses/by/4.0/), which permits unrestricted use, distribution, and reproduction in any medium, provided you give appropriate credit to the original author(s) and the source, provide a link to the Creative Commons license, and indicate if changes were made. 
complement $E^{\prime}=A / \phi^{*}(E)$ (obtained by composing the embedding $C \rightarrow A$ with the projection map) and $A$ is isogenous to the product $E \times E^{\prime}$ with kernel isomorphic to $\mathbb{Z} / n \mathbb{Z} \oplus \mathbb{Z} / n \mathbb{Z}$. We say that $J(C)$ is $(n, n)$-split. The (coarse) moduli space we wish to parametrize is the space $\widetilde{\mathcal{L}}_{n}$ of pairs $(C, E)$ related by an optimal map of degree $n>1$. (Here, optimal means that the map does not factor through an unramified cover of $E$.) It is a double cover of the moduli space $\mathcal{L}_{n}$ of genus 2 curves $C$ whose function field has an elliptic subfield of degree $n$; this latter locus is a hypersurface in $\mathcal{M}_{2}$, the moduli space of genus 2 curves.

Classically, hyperelliptic curves with split Jacobians were studied in the guise of the reduction of abelian integrals to elliptic integrals by algebraic transformations, going as far back as Legendre and Jacobi, who essentially gave a complete description of the moduli space for $n=2$. The generic family of curves $C$ for $n=3$ was worked out by Hermite, Goursat, Burkhardt, Brioschi and Bolza, and $n=4$ by Bolza. We refer the reader to [15] for a summary of this classical literature. Kuhn [16] revisited this topic, giving a combinatorial description in terms of the branch points of the map $\phi$ as well as the hyperelliptic involution $\iota$ on $C$, and adapting some examples to positive characteristic. This approach has led to fairly explicit descriptions of $\widetilde{\mathcal{L}}_{n}$ for $2 \leq n \leq 5$ by Shaska and others [23, 26-28]. From an arithmetic standpoint, reducible Jacobians for $2 \leq n \leq 4$ have been studied in $[2,3,5]$. Frey and Kani [8, 9] have also studied such covers for general $n$ by looking at pairs of elliptic curves $E$ and $E^{\prime}$ such that there is an Galois equivariant antiisometry between their $n$-torsion subschemes. Their description connects $\widetilde{\mathcal{L}}_{n}$ to several interesting arithmetic questions and applications.

In this paper, we will take a rather different strategy to compute the moduli spaces $\widetilde{\mathcal{L}}_{n}$, which does not focus on the map $\phi: C \rightarrow E$ and its ramification locus. The key fact upon which our method rests is that a genus 2 curve $C$ has a map of degree $n$ to an elliptic curve $E$ if and only if the point in $\mathcal{A}_{2}$ corresponding to its Jacobian $J(C)$ lies on the Humbert surface $\mathcal{H}_{n^{2}}$ (see [25], for instance). In other words, under the birational morphism $\mathcal{M}_{2} \rightarrow \mathcal{A}_{2}$, the image of $\mathcal{L}_{n}$ is (birationally) identified with the Humbert surface for discriminant $n^{2}$. Recall that for a discriminant $D$, the Humbert surface $\mathcal{H}_{D}$ describes principally polarized abelian surfaces which have real multiplication by the quadratic ring of discriminant $D$. Its double cover is the Hilbert modular surface $Y_{-}(D)$ which parametrizes pairs $(A, \iota)$, where $A$ is a principally polarized abelian surface, and $\iota: \mathcal{O}_{D} \rightarrow \operatorname{End}(A)$ is a ring homomorphism. Note that the reason $Y_{-}(D) \rightarrow \mathcal{H}_{D}$ is a double cover is because (generically) there are two choices for the action of $\sqrt{D}$.

In [7], a method was laid out to compute explicit models for these Hilbert modular surfaces $Y_{-}(D)$, relying on the computation of moduli spaces of suitable elliptic K3 surfaces, which are related to the abelian surfaces via a Shioda-Inose structure. In that paper, equations were given for rational models for the surfaces $Y_{-}(D)$, for the thirty fundamental discriminants $D$ such that $1<D<100$. These Hilbert modular surfaces are coarse moduli spaces for principally polarized abelian surfaces with real multiplication by the ring of integers $\mathcal{O}_{D}$ of $\mathbb{Q}(\sqrt{D})$.

In this paper, we will extend this method to compute the Humbert surfaces for square discriminants $D=n^{2}$, and our desired moduli space $\widetilde{\mathcal{L}}_{n}$ [which is birational to $Y_{-}\left(n^{2}\right)$ ], for $2 \leq n \leq 11$. To do so requires several ideas beyond those of [7]. First, we generalize the set-up and theorems in [7] to the case of non-fundamental discriminants. This 
allows us to compute $Y_{-}\left(n^{2}\right)$ as a moduli space of K3 surfaces, but does not elucidate its structure as a moduli space of $(C, E)$. In order to do so, we first describe explicitly a normalized tautological family of genus 2 curves over the moduli space, using the method of certifying eigenforms for real multiplication from [19]. Finally, we describe a new method involving integration of eigenforms to recover the elliptic curves $E_{1}$ and $E_{2}$ associated to a given $C$.

Our models agree with those in the literature for $2 \leq n \leq 5$, and we go well beyond the previous state of the art with the higher values of $n$. In doing so, we exhibit nonrational surfaces $\widetilde{\mathcal{L}}_{n}$ : the surfaces $\widetilde{\mathcal{L}}_{6}$ and $\widetilde{\mathcal{L}}_{7}$ are elliptic $\mathrm{K} 3$, the next three $\widetilde{\mathcal{L}}_{8}, \widetilde{\mathcal{L}}_{9}$ and $\widetilde{\mathcal{L}}_{10}$ are honestly elliptic, whereas $\widetilde{\mathcal{L}}_{11}$ is a surface of general type. The geometric classification of these surfaces was studied in [13,21], but explicit algebraic models were not known before this work. We anticipate that our algebraic models for these moduli spaces will make it easy to carry out explicit arithmetic and geometric investigations. For instance, one can immediately apply our formulas to produce examples or even families of elliptic curves with anti-isometric $n$-torsion Galois representations.

The plan of the paper is as follows: in Sect. 2, we prove the necessary generalizations of the results from [7]. Each of the following sections treats a separate square discriminant. We outline the parametrization of the moduli space of elliptic K3 surfaces, and a sequence of "elliptic hops" converting to an elliptic fibration with $E_{8}$ and $E_{7}$ fibers. This allows us to compute the map to $\mathcal{A}_{2}$. We can then write down the explicit double cover giving a model of $\widetilde{\mathcal{L}}_{n}=Y_{-}\left(n^{2}\right)$. We carry out some basic geometric analysis of the moduli space, and note any obvious arithmetic curves on it. We also provide a tautological family of genus 2 curves over $\widetilde{\mathcal{L}}_{n}$, as well as the $j$-invariants of both the associated elliptic curves ${ }^{1} E_{1}$ and $E_{2}$.

The auxiliary computer files for this paper, containing various formulas and computations omitted here for lack of space, are available from http://arxiv.org/abs/1412.2849. To access these, download the source file for the paper. This will produce not only the LaTeX file for this paper, but also the computer code.

For the computations involved in our work, we made frequent use of the computer algebra systems pari-gp, Magma, Maxima and Sage. We thank Nils Bruin, Henry Cohn, Noam Elkies, Gerard van der Geer, David Gruenewald, Curt McMullen, Ronen Mukamel, Dan Petersen, Matthias Schütt and the anonymous referee for helpful comments. This work was supported in part by NSF grant DMS-0952486, and by a grant from the MIT Solomon Buchsbaum Research Fund.

\section{Hilbert modular surfaces for square discriminants}

In this section, we generalize the main theorems of [7] to the case of non-fundamental discriminant $D$, which is a positive integer congruent to 0 or 1 modulo 4 . In particular, this paper will deal with the case of square discriminant: $D=n^{2}$, in which case the ring in question is an order in a split quadratic algebra (i.e. $\mathbb{Q} \oplus \mathbb{Q}$ ), rather than a real quadratic field. We first briefly go over the basic setup for this particular case, since the typical case considered in the literature is that of non-square discriminant. For more background on Hilbert modular surfaces see [30], and for a quick introduction to the

${ }^{1}$ We can make a uniform choice of one of these, so that the family of $\left(C, E_{1}\right)$ is really the tautological family over the moduli space $\widetilde{\mathcal{L}}_{n}$. 
calculations of this section, we refer the reader to [24]. The notes [6] also contain an excellent description of the theory of abelian varieties with extra endomorphisms, and the connection with $\mathrm{K} 3$ surfaces which was exploited in $[7,17]$ and is also used here.

\subsection{The quadratic ring of discriminant $D$}

Let $D=n^{2}$, and $\mathcal{O}_{D}$ the quadratic ring of discriminant $D$. Concretely, we can write

$$
\mathcal{O}_{D}=\left\{(x, y) \in \mathbb{Z}^{2}: x \equiv y \bmod n\right\} .
$$

with componentwise addition and multiplication. It has a $\mathbb{Z}$-basis $\left\{e_{1}, e_{2}\right\}$ given by $e_{1}=1=(1,1)$ and $e_{2}=(n, 0)$. The two embeddings $\sigma_{1}, \sigma_{2}$ of $K=\mathcal{O}_{D} \otimes \mathbb{Q} \cong \mathbb{Q} \oplus \mathbb{Q}$ into $\mathbb{R}$ are given by taking the first or second components and composing with $\mathbb{Q} \hookrightarrow \mathbb{R}$. There are four square roots of $D$ in $\mathcal{O}_{D}$, namely $\pm n= \pm(n, n)$ and $\pm(n,-n)$. If we label $\sqrt{D}=(n,-n)$, we see that 1 and $(D+\sqrt{D}) / 2=\left(\left(n^{2}+n\right) / 2,\left(n^{2}-n\right) / 2\right)$ also form a $\mathbb{Z}$ -basis, just as in the case of non-square discriminant. The involution which changes $\sqrt{D}$ to $-\sqrt{D}$ is that which switches the two factors; i.e. takes $(x, y)$ to $(y, x)$. The trace map takes $(x, y)$ to $x+y$, and the matrix of the trace form with respect to $e_{1}$ and $e_{2}$ is

$$
\left(\begin{array}{cc}
2 & n \\
n & n^{2}
\end{array}\right)
$$

which has discriminant $n^{2}=D$. The dual lattice $\mathcal{O}_{D}^{*}$ with respect to the trace form has basis $f_{1}=(1,0)$ and $f_{2}=\frac{1}{n}(1,-1)$. (In fact, for a general discriminant, $\mathcal{O}_{D}^{*}$ equals $\frac{1}{\sqrt{D}} \mathcal{O}_{D}$.) The discriminant group $\mathcal{O}_{D}^{*} / \mathcal{O}_{D}$ is easily calculated to be $\mathbb{Z} / n^{2} \mathbb{Z}$ if $n$ is odd, and $\mathbb{Z} / 2 \mathbb{Z} \oplus \mathbb{Z} /\left(n^{2} / 2\right) \mathbb{Z}$ if $n$ is even.

\subsection{The setup for non-fundamental discriminants}

We now adapt Sect. 3 and 4 of [7] to the setting of non-fundamental discriminant. The outline of the method is as follows.

1. The Hilbert modular surface $Y_{-}(D)$ is the coarse moduli space of $(A, \iota)$ where $A$ is a principally polarized abelian surface, and $\iota: \mathcal{O}_{D} \rightarrow \operatorname{End}(A)$ is a homomorphism. The complex manifold $Y_{-}(D)_{\mathbb{C}}$ can be obtained by compactifying $\mathrm{SL}_{2}\left(\mathcal{O}_{D}, \mathcal{O}_{D}^{*}\right) \backslash \mathcal{H}^{2}$.

2. There is a map $Y_{-}(D) \rightarrow \mathcal{A}_{2}$ which simply takes the ppas $A$. The image $\mathcal{H}_{D}$ is the Humbert surface of discriminant $D$ in $\mathcal{A}_{2}$, and the map from the Hilbert modular surface has degree 2 . The key first step is to realize $\mathcal{H}_{D}$ as a moduli space of elliptic K3 surfaces, polarized by a particular lattice $L_{D}$ of rank 18 and discriminant $-D$. We explicitly parametrize this moduli space $\mathcal{M}_{L_{D}}$, and compute the universal $\mathrm{K} 3$ family over it.

3. We then find a different elliptic fibration on the (family of) K3 surfaces, with reducible fibers of type II* and III*. This is accomplished by a sequence of "elliptic hops", which reflect 2- and 3-neighbor steps at the lattice level (see [7, Section5] or [18, AppendixA]). By the main result of [17], from the final Weierstrass equation we may read out the map $\mathcal{M}_{L_{D}} \rightarrow \mathcal{A}_{2}$, which is given in terms of Igusa-Clebsch invariants (coordinates on $\mathcal{A}_{2}[14]$ ).

4. So far, we have an explicit birational model of $\mathcal{H}_{D}$. To get to $Y_{-}(D)$, we must identify the correct double cover. We first pin down the geometric branch locus as a union 
of certain modular curves (by a result of Hausmann), and interpret these curves in terms of the K3 moduli space (the Picard group jumps in a predictable manner). Finally, we find the correct arithmetic twist by point-counting and matching characteristic polynomials of Frobenius on the abelian and K3 sides.

The first part of [7, Section3] outlines the proof that $\mathrm{SL}_{2}\left(\mathcal{O}_{D}, \mathcal{O}_{D}^{*}\right) \backslash \mathcal{H}^{2}$ is the coarse moduli space of principally polarized abelian surfaces with real multiplication by $\mathcal{O}_{D}$. The same proof goes through for arbitrary discriminant. Of course, for $D=n^{2}$, usually it is more convenient to understand a ppas with real multiplication by $\mathcal{O}_{D}$ in terms of pairs of elliptic curves which have degree $n$ maps from a common genus 2 curve, as described in the introduction. This is the approach taken in $[4,13,21]$.

Proposition 1 Let $A$ be a principally polarized abelian surface with $\operatorname{End}(A) \cong \mathcal{O}_{D}$. Then $\mathrm{NS}(A) \cong \operatorname{End}(A)$. The lattice $\mathrm{NS}(A)$ has a basis with Gram matrix

$$
\left(\begin{array}{cc}
2 & D \\
D & \left(D^{2}-D\right) / 2
\end{array}\right)
$$

of signature $(1,1)$ and discriminant $-D$.

The proof of [7] goes through with minor changes, noting that the Rosati involution on $K$ must be the identity, since the other element of the Galois group (interchange of the factors in the square-discriminant case) is not a positive involution.

The proof of the next proposition is unchanged from the original.

Proposition 2 There is a primitive embedding, unique up to isomorphism, of the lattice $\mathcal{O}_{D}$ into $U^{3}$. Let $T_{D}$ be the orthogonal complement of $\mathcal{O}_{D}$ in $U^{3}$. Then there is a primitive embedding, unique up to isomorphism, of $T_{D}$ into the K3 lattice $\Lambda$.

The main theorem of that section identifies the Humbert surface with a moduli space of $\mathrm{K} 3$ surfaces lattice-polarized by $L_{D}:=E_{8}(-1)^{2} \oplus \mathcal{O}_{D}$. Let $L$ be the lattice $U \oplus E_{8}(-1) \oplus E_{7}(-1)$.

Theorem 3 Let $\mathcal{F}_{L_{D}}$ be the moduli space of K3 surfaces that are lattice polarized by $L_{D}$. Then the isomorphism $\phi: \mathcal{F}_{L} \rightarrow \mathcal{A}_{2}$ of [17] induces a birational surjective morphism $\mathcal{F}_{L_{D}} \rightarrow \mathcal{H}_{D}$

Its proof relies on a key proposition involving the lattice embedding $L_{D} \hookrightarrow L$, where $L=U \oplus E_{8}(-1) \oplus E_{7}(-1)$ and $L_{D} \cong E_{8}(-1)^{2} \oplus \mathcal{O}_{D}$ is the orthogonal complement of $T_{D}$ in $\Lambda$, uniquely described by the proposition above. The proof of the key proposition and the main theorem go through without any changes.

We must next show that the branch locus of the map $Y_{-}(D) \rightarrow \mathcal{H}_{D}$ corresponds to a divisor $Z$ in the moduli space of $\mathcal{M}_{L_{D}}$ of $\mathrm{K} 3$ surfaces lattice polarized by $L_{D}$, such that the rank of the K3 surface corresponding to a generic point on $Z$ jumps to 19 , and the discriminant of the Picard group is $D / 2$ or $2 D$. In [7], the argument used the fact that $D$ was fundamental; we give a more general proof here, valid for arbitrary discriminant. 
First, we observe as in [7] that the branch locus consists of a union of specific modular curves. Let us consider the birational models $\Gamma \backslash \mathcal{H}^{2}$ for $Y_{-}(D)\left(\right.$ where $\Gamma=\mathrm{SL}_{2}\left(\mathcal{O}_{D}, \mathcal{O}_{D}^{*}\right)$ ) and $\mathrm{Sp}_{4}(\mathbb{Z}) \backslash \mathcal{S}_{2}$ for $\mathcal{A}_{2}$, with $\mathcal{S}_{2}$ being the Siegel upper half space for genus 2 . The twoto-one map $\Gamma \backslash \mathcal{H}^{2} \rightarrow \mathrm{Sp}_{4}(\mathbb{Z}) \backslash \mathcal{S}_{2}$ factors through $(\Gamma \cup \Gamma \sigma) \backslash \mathcal{H}^{2}$, where $\sigma$ is the interchange of coordinates on $\mathcal{H}^{2}$. The branch locus is the fixed point set of $\sigma$ on $\Gamma \backslash \mathcal{H}^{2}$.

Proposition 4 (Hausmann [12]) The one-dimensional part of the fixed point set of $\sigma$ on $\Gamma \backslash \mathcal{H}^{2}$ is the union of the modular curves $F_{w}$, where $w$ ranges over $\{1, D\}$ if $D$ is odd, and over $\{1,4, D / 4, D\}$ if $D$ is even.

Next, we analyze what happens to the K3 surfaces along these modular curves.

Proposition 5 Let $N$ be a natural number, and let $C$ be any component of the inverse image of a modular curve $F_{N}$, under the birational surjective morphism $\phi_{D}: \mathcal{F}_{L_{D}} \rightarrow \mathcal{H}_{D}$. The K3 surface corresponding to a generic point of $C$ has Néron-Severi group of rank 19 and discriminant $2 \mathrm{~N}$.

Proof By [24], the generic point on any component of a modular curve $F_{N}$ corresponds to an abelian surface $A$ whose ring of endomorphisms is a quaternionic order $R$ of discriminant $N^{2}$. By [11], the Néron-Severi group of $A$ and the endomorphism ring are connected by the relation $R=\mathcal{C}^{+}\left(\frac{1}{2} \mathrm{NS}(A)\right)$, the even part of the associated Clifford ring. From this, it is easy to compute that $\mathrm{NS}(A)$ must have rank 3 and discriminant $2 N$. By the Shioda-Inose structure, the Picard group of the associated K3 surface must have rank 19 and discriminant $2 N$.

Therefore, the part of the branch locus corresponding to the curve $F_{D}$ (and $F_{D / 4}$ if $D$ is even) corresponds to a sublocus of K3 surfaces having Picard group of discriminant $2 D$ (respectively $D$ / 2). Section 4 of [7] explains how to correctly identify the branch locus and the arithmetic twist; we will not restate the method or proofs here.

The discussion for the curves $F_{1}$ (and $F_{4}$ if $D$ is even) was omitted from [7]; we address it here.

Proposition 6 Let $X$ be an elliptic K3 surface with a reducible fiber of type $\mathrm{II}^{*}$ and another of type III*. Furthermore, assume that $X$ has a section of height $D / 2$, for some natural number $D$ congruent to 0 or 1 modulo 4. Then NS(X) cannot be a lattice of rank 19 and discriminant 2 or 8 .

Proof First, assume that $X$ has no other reducible fibers. Then, in particular, there cannot be a torsion section: such a section would have height 0 , which is impossible to obtain with only two reducible fibers of type $E_{8}$ and $E_{7}$. Therefore, if $\operatorname{NS}(X)$ had discriminant 2, the Mordell-Weil lattice would be positive definite of rank 2 and discriminant 1 . The Hermite constant in dimension 2 is $2 / \sqrt{3}$, so this would imply that there is a section of height at most $2 / \sqrt{3} \approx 1.155$, which is impossible with the fiber configuration (the best we can do is $4-4 / 3=8 / 3>2.66$ ). Similarly, if NS $(X)$ had discriminant 8 , we would need a section of height $4 / \sqrt{3} \approx 2.309$, which is still forbidden. Note that we did not use the assumption of a section of height $D / 2$. For the case when $X$ could have 
additional reducible fibers, we have to do some additional analysis, but a similar proof applies.

Going back to our situation, let $\mathcal{X}$ be the family of $\mathrm{K} 3$ surfaces lattice polarized by $L_{D}$, over the base $\mathcal{M}_{L_{D}}$ (the coarse moduli space). In the third step of the method described above, we find a different elliptic fibration on the K3 surface $\mathcal{X}_{v}$ (uniformly over $v$ on

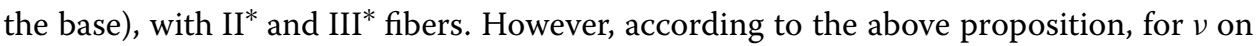
one of the curves $C$ lying over $F_{1}$ or $F_{4}$, we cannot have such an elliptic fibration on $X_{\nu}$. Therefore, the Weierstrass equation for the resulting $\mathrm{II}^{*}$, III* fibration must be undefined (or not minimal, or one of these fibers must degenerate further) for $v$ on such a curve $C$. This is reflected in the denominators of the expressions for the Weierstrass coefficients and of the Igusa-Clebsch invariants, as well as the (numerator of the) invariant $I_{10}$. Therefore, by adding their irreducible factors to that of the list for discriminant $2 D$ (and $D / 2$ when $D$ is even), we can then continue with Step 4 of [7].

The remaining assertions and proofs of Section 4 of [7] go through without any change. Therefore, we may now proceed with the computation of the Hilbert modular surfaces $Y_{-}\left(n^{2}\right)$ for small values of $n$. Here, we describe these surfaces for $n$ up to 11 . The cases $2 \leq n \leq 5$ have been treated in the previous literature on the subject, although by using completely different methods. The cases $6 \leq n \leq 11$ show that one can quickly go beyond the current state of the art using our new techniques. For each $n$ treated in this paper, we also describe a tautological family of genus 2 curves over the moduli space, whose Jacobians are $(n, n)$-isogenous to a pair of elliptic curves, and we give the $j$-invariants of these elliptic curves. ${ }^{2}$ To accomplish this, we use the Eigenform Location Algorithm of [19] to produce a tautological family of normalized genus 2 curves (i.e. for which the eigenforms for real multiplication are given by $\mathrm{d} x / y$ and $x \mathrm{~d} x / y$ ), along with a method which involves integrating an eigenform. In principle, we can even give the two maps of degree $n$ from the genus 2 curve to the elliptic curves; we indicate how this is done in the case $n=3$. There is no serious obstruction to computing these modular surfaces for $n \geq 12$, though of course the computations will get more challenging for large $n$.

We list the geometric type of the surfaces treated here in the following table. For comparison with the literature, we note that Hermann [13] described the geometric classification of modular surfaces $Y_{n, \epsilon}$ of discriminant $n^{2}$, which is the quotient of $\mathcal{H}^{2}$ by an appropriate subgroup of $\mathrm{SL}_{2}(\mathbb{Z} / n \mathbb{Z})^{2}$. A different proof was given by Kani and Schanz [21], who also corrected a typo in [13], and described the connection with the moduli space of pairs of elliptic curves $E_{1}, E_{2}$ with an isomorphism on the $n$-torsion $E_{1}[n] \cong E_{2}[n]$. The index $\epsilon \in(\mathbb{Z} / n \mathbb{Z})^{\times}$is the factor which multiplies the determinant of the Weil pairing under this isomorphism. See also [4] for a nice overview and connections to modular forms. For our particular situation, $\epsilon=-1$ as the isomorphism is an anti-isometry (see [9]), and we can read off the corresponding results from the above papers (note that the isomorphism type only depends on the square class of $\epsilon$ in $\left.(\mathbb{Z} / n \mathbb{Z})^{\times}\right)$.

\footnotetext{
${ }^{2}$ More specifically, when the moduli space $Y_{-}\left(n^{2}\right)$ is rational, we give the $j$-invariants in terms of the parameters on the moduli space. For the non-rational moduli spaces, we give $j_{1}+j_{2}$ and $j_{1} j_{2}$ in terms of the parameters on the (rational) Humbert surface $\mathcal{H}_{n^{2}}$, and verify that $j_{1}-j_{2}$ generates the function field of its double cover $Y_{-}\left(n^{2}\right)$.
} 


\begin{tabular}{ll}
\hline $\boldsymbol{n}$ & Geometric type \\
\hline 2 & Rational \\
3 & Rational \\
4 & Rational \\
5 & Rational \\
6 & Elliptic K3 \\
7 & Elliptic K3 \\
8 & Honestly elliptic \\
9 & Honestly elliptic \\
10 & Honestly elliptic \\
11 & General type \\
\hline
\end{tabular}

\section{Discriminant 4}

\subsection{Parametrization}

We would like a family of $\mathrm{K} 3$ surfaces lattice polarized by $L_{4}$. It is easy to see that $L_{4} \cong U \oplus E_{8} \oplus E_{7} \oplus A_{1}$. We now reverse-engineer Tate's algorithm to find elliptic K3 surfaces with reducible fibers of types $E_{8}, E_{7}$ and $A_{1}$ at $t=\infty, 0$ and 1 respectively.

A general elliptic K3 surface with section has the form

$$
y^{2}=x^{3}+a(t) x^{2}+b(t) x+c(t),
$$

with $a, b, c$ being polynomials in $t$ of degree $4,8,12$ respectively. In order to have an $E_{8}$ fiber at $t=\infty$, we may assume (after shifting $x$ suitably) that $a$ has degree at most 2. Similarly, the $E_{7}$ fiber at $t=0$ allows us to assume that $a=k t^{2}$, for some constant $k$. These normalizations involve shifting $x$ by a linear combination of $1, t, t^{3}$ and $t^{4}$. Finally, since there is an $A_{1}$ fiber at $t=1$, we may shift $x$ by a suitable multiple of $t^{2}$, to make $b(t)$ and $c(t)$ divisible by $t-1$ and $(t-1)^{2}$ respectively. The final result is that the Weierstrass equation has the form

$$
y^{2}=x^{3}+e t^{2} x^{2}+f t^{3}(t-1) x+g t^{5}(t-1)^{2} .
$$

Since we must quotient by the Weierstrass scaling of $x$ and $y$, we see that the resulting moduli space is a weighted projective space $\mathbb{P}(2: 4: 6)$ in the coordinates $e, f, g$.

\subsection{Map to $\mathcal{A}_{2}$ and equation of $\tilde{\mathcal{L}_{2}}$}

To compute the map to $\mathcal{A}_{2}$, we put the surface in the standard form described in [17]. Here, it merely involves shifting $x$ so that the coefficient of $x^{2}$ is zero, and then rescaling so that the coefficient of $x t^{3}$ is -1 . We obtain the Weierstrass equation

$$
y^{2}=x^{3}-t^{3}\left(\frac{3 f-e^{2}}{3} t-1\right) x+t^{5}\left(f g t^{2}-\frac{54 g+9 e f-2 e^{3}}{27} t+\frac{3 g+e f}{3 f}\right) .
$$

We may now read out the Igusa-Clebsch invariants, which are functions of $r=f / e^{2}$ and $s=g / e^{3}$. We obtain 


$$
\begin{aligned}
I_{2} & =8(3 s+r) / r \\
I_{4} & =-4(3 r-1), \\
I_{6} & =-4\left(6 r s-8 s+5 r^{2}-2 r\right) / r \\
I_{10} & =4 r s .
\end{aligned}
$$

By considering the possible ways the rank could jump and give K3 surfaces whose Néron-Severi lattices have the appropriate discriminant, we obtain the following list of possible factors for the branch locus:

$$
r, \quad s, \quad\left(4 s-r^{2}\right), \quad\left(9 s-3 r^{2}+r\right), \quad\left(27 s^{2}+36 r s-s-16 r^{3}+8 r^{2}-r\right) .
$$

An arithmetic verification as in [7] pins down the correct double cover.

Theorem 7 A birational model for the surface $\widetilde{\mathcal{L}}_{2}$ (equivalently, for $Y_{-}(4)$ ) is given by

$$
z^{2}=-r\left(27 s^{2}+36 r s-s-16 r^{3}+8 r^{2}-r\right)
$$

It is a rational surface. The Humbert surface is birational to the $(r, s)$-plane. In these coordinates, the Igusa-Clebsch invariants of a point on the moduli space are given by the formulas in (2) above.

This is a conic bundle over $\mathbb{P}_{r}^{1}$. Setting $s=0$ makes this expression a square, so in fact this conic bundle has a section, and is a rational surface over the base field $\mathbb{Q}$. To parametrize it, we complete the square by letting $z=m s+r(4 r-1)$. Solving for $s$, we obtain

$$
s=-\frac{r(8 m r+36 r-2 m-1)}{27 r+m^{2}}, \quad z=\frac{r\left(108 r^{2}-4 m^{2} r-36 m r-27 r+m^{2}+m\right)}{27 r+m^{2}} .
$$

\subsection{Comparison with previous formulae}

The classical formulae over an algebraically closed field are described in [27], for instance. We start with a genus 2 curve

$$
y^{2}=x^{6}-s_{1} x^{4}+s_{2} x^{2}-1
$$

which has two obvious maps to elliptic curves. The group $D_{12}$ acts on the family of such Weierstrass equations as follows: its two generators take $\left(x, y, s_{1}, s_{2}\right)$ to $\left(\zeta_{6} x, y, s_{1} \omega, s_{2} \omega^{2}\right)$ or to $\left(1 / x, i y / x^{3}, s_{2}, s_{1}\right)$, where $\zeta_{6}$ is a primitive sixth root of unity, $\omega=\zeta_{6}^{2}$ and $i=\sqrt{-1}$. The invariants of this action on the polynomial ring generated by the parameters $s_{1}$ and $s_{2}$ are $u=s_{1} s_{2}$ and $v=s_{1}^{3}+s_{2}^{3}$. Comparing Igusa-Clebsch invariants, we obtain the relation between our coordinates $r, s$ above and $u, v$.

$$
\begin{aligned}
& r=\frac{s_{1}^{2} s_{2}^{2}-4 s_{1}^{3}-4 s_{2}^{3}+18 s_{1} s_{2}-27}{4\left(s_{1} s_{2}-9\right)^{2}}=\frac{u^{2}-4 v+18 u-27}{4(u-9)^{2}} \\
& s=\frac{2\left(s_{1}^{2} s_{2}^{2}-4 s_{1}^{3}-4 s_{2}^{3}+18 s_{1} s_{2}-27\right)}{\left(s_{1} s_{2}-9\right)^{3}}=\frac{2\left(u^{2}-4 v+18 u-27\right)}{(u-9)^{3}}
\end{aligned}
$$

with inverse

$$
\begin{aligned}
& u=(9 s+8 r) / s, \\
& v=2\left(27 s^{2}+36 r s-32 r^{3}+8 r^{2}\right) / s^{2} .
\end{aligned}
$$


The branch locus, up to squares, equals $\left(v^{2}-4 u^{3}\right)=\left(s_{1}^{3}-s_{2}^{3}\right)^{2}$. Recall that interchanging $s_{1}$ and $s_{2}$ changes the equation of the genus 2 curve by $x \rightarrow 1 / x$, and so switches the two elliptic subfields. So it agrees with the classical double cover. Another explicit way to see this is through the $j$-invariants, which we compute next.

\subsection{Tautological genus 2 curve and elliptic curves}

Over the moduli space $Y_{-}(4)$ (or $\widetilde{\mathcal{L}}_{2}$ ), it is possible to write down a tautological family of genus 2 curves with $(2,2)$ reducible Jacobian. In terms of $r, s, z$ above, the sextic defining the family is

$$
\begin{aligned}
y^{2}= & x^{6}+(9 s+8 r) x^{4}-\left(8 r z-27 s^{2}-36 r s+32 r^{3}-8 r^{2}\right) x^{2} \\
& -s\left(8 r z-27 s^{2}-36 r s+32 r^{3}-8 r^{2}\right) .
\end{aligned}
$$

The $j$-invariants corresponding to the two elliptic subfields are

$$
\pm \frac{128(4 r-1)}{s^{2}} z-\frac{64\left(36 r s-s-32 r^{3}+16 r^{2}-2 r\right)}{s^{2}},
$$

which again shows that the involution switching the $j$-invariants is the one corresponding to the double cover.

Since the Hilbert modular surface is rational with parameters $r, m$, we may further simplify the tautological family above.

Theorem 8 In terms of the parameters $(r, m)$ on the Hilbert modular surface, a tautological family of genus 2 curve with $(2,2)$-reducible Jacobian is given by

$$
y^{2}=x^{6}-(2 m+3) x^{4}-(36 r-4 m-3) x^{2}+4(2 m+9) r-2 m-1 .
$$

\subsection{Special loci}

We now describe some special curves on the Hilbert modular surface.

1. The genus 0 curve $r=0$ is part of the branch locus; it is also part of the product locus, i.e. the boundary $\left(\mathcal{A}_{2} \backslash \mathcal{M}_{2}\right) \cap \mathcal{H}_{4}$ which corresponds to products of elliptic curves rather than Jacobians of genus 2 curves.

2. For the curves $(r, s, z)=(r, 0, \pm r(4 r-1))$, the invariant $I_{10}=0$. They correspond to degenerations of the genus 2 curve to a rational curve.

3. The curve $s=r^{2} / 4$ on the Humbert surface pulls back to a genus 0 curve on $Y_{-}(4)$, isomorphic to $\mathbb{P}^{1}$. It is a modular curve, corresponding to Jacobians with endomorphisms by a (split) quaternion algebra. The minimal degree of the isogeny between the two elliptic curves we have associated above is 3 . This curve is the same as that defined in [27, formula (11)].

4. The curve $r=1 / 4$ also lifts to a $\mathbb{P}^{1}$ on the Hilbert modular surface, corresponding to the modular curve for which $j_{1}=j_{2}$.

5. The curve $r=-1 / 2, z= \pm(27 s-2) / 18$ corresponds to one of the $j$-invariants being 0 (i.e. having $\mathrm{CM}$ by $\mathbb{Z}[\omega]$ ). 
6. The curve $27 s^{2}+36 r s-s-16 r^{3}+8 r^{2}-r=0$ has genus 0 and is part of the branch locus. It is parametrized by $(r, s)=\left(\left(t^{2}-1\right) / 12,(t-2)^{2}(t+1) / 54\right)$ and corresponds to the $j$-invariants being equal.

7. The curve $3375 s^{2}-1440 r s-152 s-64 r^{3}+48 r^{2}-12 r+1=0$ lifts to a genus 0 curve on the Hilbert modular surface; its parametrization on the Humbert surface is given by $(r, s)=\left(\left(t^{2}-1\right) / 60,(t+4)^{3} / 3375\right)$. It corresponds to the elliptic curves being 2 -isogenous.

8. There are also several simple non-modular curves, for example the curves $s=1 / 27$, $s=2 / 27$, and $s=r^{2} / 3-r / 9$. These lift to genus 0 curves (in fact, isomorphic to $\mathbb{P}^{1}$ ) on $Y_{-}(4)$.

Remark 9 Since the Hilbert modular surface is rational, in fact we have a 2-parameter family of genus 2 curves with $(2,2)$-split Jacobian given by the equation above, and we can specialize it to produce infinitely many examples of 1-parameter families of such genus 2-curves.

Remark 10 To check the statement that elliptic curves corresponding to points on a specific curve on $\widetilde{\mathcal{L}}_{2}$ are (say) 2 -isogenous, one can simply substitute in the $j$-invariants into the appropriate classical modular polynomial. Another way to verify that such a curve on the Hilbert modular surface is modular is to check that the Picard number of the elliptic K3 surface jumps (for instance, due to an extra reducible fiber, or an extra section). From now on, we shall make such statements without further justification; the interested reader may carry out the (easy) check.

\section{Discriminant 9}

\subsection{Parametrization}

Next, we describe degree 3 elliptic subfields, which were treated by Shaska in [26]. A simple family of elliptic K3 surfaces which will allow us to recover this moduli space is that with $E_{8}$ and $A_{8}$ fibers. As before, we put the $E_{8}$ fiber at $\infty$, letting us write the Weierstrass equation as

$$
y^{2}=x^{3}+a(t) x^{2}+2 b(t) x+c(t)
$$

with $a, b, c$ polynomials of degrees $2,4,7$ respectively. Assuming the $A_{8}$ fiber is at $t=0$, and shifting $x$ by a suitable quadratic polynomial, we can rewrite the Weierstrass equation as

$$
y^{2}=x^{3}+\left(a_{0}+a_{1} t+a_{2} t^{2}\right) x^{2}+2 t^{3}\left(b_{0}+b_{1} t\right) x+t^{6}\left(c_{0}+c_{1} t\right) .
$$

This surface generically has an $A_{5}$ fiber at $t=0$. To ensure an $A_{8}$ fiber, we need three more orders of vanishing for the discriminant at $t=0$. Since we need the components of the $\mathrm{I}_{9}$ fiber to be defined over the ground field, the coefficient $a_{0}$ is a nonzero square; by scaling $x$ we may assume it is 1 . Similarly, by scaling $t$, we can arrange $c_{0}=c_{1}$.

The three orders of vanishing successively yield $b_{0}=c_{0}^{2}, b_{1}=b_{0}\left(a_{1}+1\right) / 2$, and $a_{2}=\left(a_{1}-1\right)^{2} / 4$. Relabeling $c_{0}=r$ and $a_{1}=s$, we obtain the final Weierstrass equation 


$$
y^{2}=x^{3}+\left(\frac{(s-1)^{2}}{4} t^{2}+s t+1\right) x^{2}+t^{3} r((s+1) t+2) x+r^{2} t^{6}(t+1)
$$

over the rational moduli space with parameters $r, s$.

\subsection{Map to $\mathcal{A}_{2}$ and equation of $\tilde{\mathcal{L}}_{3}$}

To compute the map to $\mathcal{A}_{2}$, we go to an elliptic fibration with $E_{8}$ and $E_{7}$ fibers via a 2-neighbor step (see [7, 18]).

In terms of Dynkin diagrams of rational curves on the K3 surface, the picture is as follows:

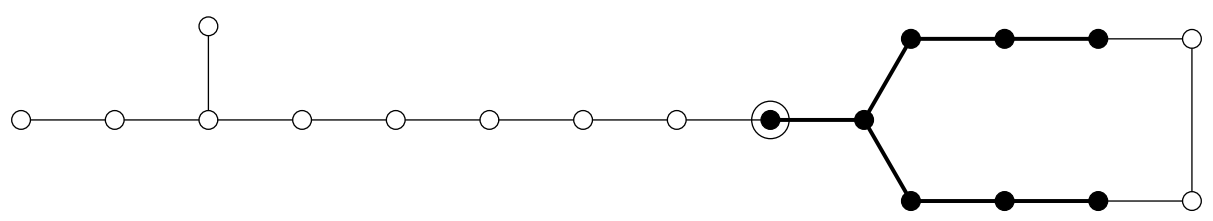

The dark vertices form a sub-diagram cutting out an $E_{7}$ fiber. The corresponding elliptic parameter (unique up to fractional linear transformations) is $w=\left(x+r t^{3}\right) / t^{4}$. Substituting $x=w t^{4}-r t^{3}$ in to the Weierstrass equation, and dividing the right hand side by $t^{8}$, we obtain a quartic in $t$, giving rise to a genus 1 curve over $\mathbb{P}_{w}^{1}$. In fact, the genus 1 fibration has a section (it is evident from the diagram above), so we may convert to the Jacobian using classical formulas (see [1], for instance). After some scaling and normalization, we arrive at a Weierstrass equation in standard form, with the following equation

$$
\begin{aligned}
Y^{2}= & X^{3}+\left(-27\left(s^{4}-4 s^{3}+6 s^{2}-48 r s-4 s+192 r+1\right) T^{4}-5184 T^{3}\right) X \\
& +15552\left(s^{2}+4 s-2\right) T^{5}+54\left(s^{6}-6 s^{5}+15 s^{4}-72 r s^{3}-20 s^{3}-432 r s^{2}\right. \\
& \left.+15 s^{2}+1080 r s-6 s+864 r^{2}-576 r+1\right) T^{6}+46656 r^{3} T^{7} .
\end{aligned}
$$

From this we may read out the Igusa-Clebsch invariants.

$$
\begin{aligned}
I_{2}= & 8\left(s^{2}+4 s-2\right) \\
I_{4}= & 4\left(s^{4}-4 s^{3}+6 s^{2}-48 r s-4 s+192 r+1\right) \\
I_{6}= & 8\left(s^{6}+2 s^{5}-21 s^{4}-40 r s^{3}+44 s^{3}+144 r s^{2}-41 s^{2}+792 r s\right. \\
& \left.+18 s-288 r^{2}-320 r-3\right) \\
I_{10}= & 2^{14} r^{3} .
\end{aligned}
$$

A similar analysis as for discriminant 4 computed the double cover of the Humbert surface giving the Hilbert modular surface.

Theorem 11 A birational model for the surface $\widetilde{\mathcal{L}}_{3}$ (equivalently, for $Y_{-}(9)$ ) is given by

$$
z^{2}=11664 r^{2}-8\left(54 s^{3}+27 s^{2}-72 s+23\right) r+(s-1)^{4}(2 s-1)^{2} .
$$

It is a rational surface. The Humbert surface is birational to the $(r, s)$-plane. In these coordinates, the Igusa-Clebsch invariants of a point on the moduli space are given by the formulas in (3) above. 
The equation above describes a conic bundle over $\mathbb{P}_{s}^{1}$. Setting $r=0$ makes the right side a square, so the conic bundle has a section. To parametrize the surface, we set $z=4 m r+(s-1)^{2}(2 s-1)$ and solve for $r$, obtaining

$$
\begin{aligned}
& r=-\frac{(s-1)^{2}(2 s-1) m+\left(54 s^{3}+27 s^{2}-72 s+23\right)}{2(m-27)(m+27)} \\
& z=-\frac{(s-1)^{2}(2 s-1)\left(m^{2}+729\right)+2\left(54 s^{3}+27 s^{2}-72 s+23\right) m}{(m-27)(m+27)} .
\end{aligned}
$$

\subsection{Tautological curve}

A tautological curve over the Hilbert modular surface (which is rational in the parameters $m, s$ above) is given by

$$
\begin{aligned}
y^{2}= & \left(x^{3}+3(3 s-1) x^{2}-\frac{2(m-27)(9 s-5)^{2}}{(m+27)}\right) \\
& \times\left(x^{3}-\frac{3(m-27)(9 s-5)^{2}}{4(m+27)} x+\frac{(m-27)(9 s-5)^{3}}{4(m+27)}\right) .
\end{aligned}
$$

Remark 12 This presentation of the curve has the feature that the eigen-differentials for the action of real multiplication by $\mathcal{O}_{9}$ (alternatively, the pullbacks of the canonical differentials from the two elliptic curves) are $d x / y$ and $x d x / y$. We say that the Weierstrass equation is normalized. ${ }^{3}$ The Weierstrass equations given in this paper and in the auxiliary files for tautological families of genus 2 curves (for each of the discriminants) will always be normalized. To check this property of being normalized amounts to a calculation using the "eigenform location" algorithm of [19] In this paper, we omit the details.

\subsection{Elliptic curves}

Next, we compute the $j$-invariants associated of the two associated elliptic curves.

\subsubsection{Calculation of $j$-invariants}

To calculate the $j$-invariants in this and other sections, we used the following computational technique. For many specializations $(r, s) \in \mathbb{Q}^{2}$, we compute the genus 2 curve from its Igusa-Clebsch invariants. We then decompose its Jacobian and find out its simple factors, using analytic techniques (using the function AnalyticJacobian in Magma; see [33]). More precisely, let $A$ be the analytic Jacobian. If the coordinates $r, s$ are chosen generically, its endomorphism ring will be $\mathcal{O}_{9}$, the quadratic ring of discriminant 9. We solve the equation $\eta^{2}=3 \eta$ in $\operatorname{End}(A)$. Then we can recover the period matrices of $E_{1}=A / \eta A$ and $E_{2}=A /(\eta-3) A$, and from there compute the two $j$-invariants. In general, these are quadratic over the base field, but their sum and product are rational. We use rational reconstruction to recover these numbers $\left(j_{1}+j_{2}\right)(r, s)$ and $\left(j_{1} j_{2}\right)(r, s)$ from floating-point approximations. Finally, we apply the above procedure to enough

3 There is still an extra degree of freedom from scaling the $x$-coordinate, and we make an arbitrary choice, designed to make the equation look "nice". 
specializations to enable us to recover $j_{1}+j_{2}$ and $j_{1} j_{2}$ as rational functions, by interpolation/linear algebra (using some upper bounds on the degree as in [32]) .

We obtain the following expressions:

$$
\begin{aligned}
j_{1}+j_{2}= & \left(2 s^{9}-17 s^{8}+64 s^{7}-324 r s^{6}-140 s^{6}+1350 r s^{5}+196 s^{5}-2097 r s^{4}\right. \\
& -182 s^{4}+17496 r^{2} s^{3}+1368 r s^{3}+112 s^{3}-23328 r^{2} s^{2}-162 r s^{2}-44 s^{2} \\
& \left.+9720 r^{2} s-198 r s+10 s-314928 r^{3}-432 r^{2}+63 r-1\right) / r^{2}, \\
j_{1} j_{2}= & \left(s^{4}-4 s^{3}+6 s^{2}+432 r s-4 s-288 r+1\right)^{3} / r^{3} .
\end{aligned}
$$

The discriminant of $T^{2}-T\left(j_{1}+j_{2}\right)+j_{1} j_{2}$ is, up to squares, the branch locus of the double cover defining the Hilbert modular surface. Solving in terms of $m$ and $s$, we have (up to interchange)

$$
\begin{aligned}
& j_{1}=\frac{4(m+27)\left(m s^{2}+27 s^{2}-2 m s+18 s+m-21\right)^{3}}{(m-27)\left(2 m s^{3}+54 s^{3}-5 m s^{2}+27 s^{2}+4 m s-72 s-m+23\right)}, \\
& j_{2}=\frac{-2(m-27)\left(m s^{2}-459 s^{2}-2 m s+486 s+m-123\right)^{3}}{(m+27)\left(2 m s^{3}+54 s^{3}-5 m s^{2}+27 s^{2}+4 m s-72 s-m+23\right)^{2}} .
\end{aligned}
$$

The above process may seem non-rigorous, but in fact can be made completely rigorous, for instance, by demonstrating the real multiplication by $\mathcal{O}_{9}$ through an explicit correspondence (as described in [32] or in more detail in the forthcoming note [20]). For reasons of space, we do not describe this method explicitly here.

\subsubsection{Calculation of the morphism}

However, we will describe another method, suggested to us by Noam Elkies, which makes use of the fact that the differentials on the genus 2 curve are normalized (i.e. are eigenforms for real multiplication).

We start with the (arbitrarily chosen) point $s=5, m=13$ on $\widetilde{\mathcal{L}}_{3}$. Rescaling the $x$-coordinate so the Weierstrass equation has integer coefficients, the genus 2 curve is given by

$$
y^{2}=\left(x^{3}+420 x-5600\right)\left(x^{3}+42 x^{2}+1120\right) .
$$

For convenience, let us instead start from the curve $C$

$$
y^{2}=\left(-5600 x^{3}+420 x^{2}+1\right)\left(1120 x^{3}+42 x+1\right)
$$

(obtained by the change of variables $x \rightarrow 1 / x, y \rightarrow y / x^{3}$ ) which has the rational point $(0,1)$. The form $x \mathrm{~d} x / y$ is a normalized differential on $C$ (since $\mathrm{d} x / y \mapsto\left(-1 / x^{2}\right) \mathrm{d} x /\left(y / x^{3}\right)=-x \mathrm{~d} x / y$ under the above transformation). Assume that the associated elliptic curve $E$ is given by

$$
y_{1}^{2}=F x_{1}^{3}+G x_{1}^{2}+H x_{1}+1
$$

where $F, G$ and $H$ are (for the moment) undetermined parameters. Also, let us assume without loss of generality that the morphism $\phi: C \rightarrow E$ which we want to determine takes $(0,1) \in C$ to $(0,1) \in E$. Note that the above model for $E$ can easily be converted to standard Weierstrass form. 
Our normalization assumption says that $\phi^{*}$ maps the canonical differential $\mathrm{d} z_{1}=\mathrm{d} x_{1} / y_{1}$ on $E$ to $\mathrm{d} z=x \mathrm{~d} x / y$ on $C$. Expanding in the formal ring about $(0,1)$ on these curves, we compute as follows:

$$
\frac{x \mathrm{~d} x}{y}=\frac{x \mathrm{~d} x}{\left(\left(-5600 x^{3}+420 x^{2}+1\right)\left(1120 x^{3}+42 x+1\right)\right)^{1 / 2}}=\left(x-21 x^{2}+903 x^{3} / 2+\cdots\right) \mathrm{d} x .
$$

Therefore

$$
z=\int x \mathrm{~d} x / y=x^{2} / 2-7 x^{3}+\cdots
$$

On the other hand, we have

$$
\frac{\mathrm{d} x_{1}}{y_{1}}=\frac{\mathrm{d} x_{1}}{\left(F x_{1}^{3}+G x_{1}^{2}+H x_{1}+1\right)^{1 / 2}}=\left(1-\frac{H}{2} x_{1}+\left(\frac{-G}{2}+\frac{3 H^{2}}{8}\right) x_{1}^{2}+\cdots\right) \mathrm{d} x_{1},
$$

So

$$
z_{1}=\int \mathrm{d} x_{1} / y_{1}=x_{1}-(H / 4) x_{1}^{2}+\cdots
$$

Now, since $\mathrm{d} z=\phi^{*}\left(\mathrm{~d} z_{1}\right)$, we have the equation $z=\phi^{*}\left(z_{1}\right)$, which we can write using the above expressions as

$$
x^{2} / 2-7 x^{3}+\cdots=\phi^{*}\left(x_{1}-(H / 4) x_{1}^{2}+\cdots\right)=x_{E}-(H / 4) x_{E}^{2}+\cdots,
$$

where $x_{E}=\phi^{*}\left(x_{1}\right)$. We can invert the formal series on the right to write

$$
x_{E}=x^{2} / 2-7 x^{3}+(H / 16+903 / 8) x^{4}+\cdots .
$$

Now, we know that $x_{E}=\phi^{*}\left(x_{1}\right)$ must be a rational function of degree 3 in $x$ (for a degree $n$ map $C \rightarrow E$, it will have degree $n)$. Since its formal expansion starts $x^{2} / 2+\cdots$, it must equal

$$
\frac{x^{2}(1+k x)}{2\left(1+m x+n x^{2}+p x^{3}\right)}
$$

for some constants $k, m, n, p$ which are also undetermined.

We expand the above rational expression in a power series about $x=0$ and match with the previous formal expression for $x_{E}$. The first few coefficients give us linear equations which can be solved for $m, n, p$ and $k$ in that order, and then we can solve the next few polynomial equations for $F, G, H$. Renormalizing the equations of $C$ and $E$, we find that the elliptic curve $E$ is given by the Weierstrass equation

$$
y_{1}^{2}=x_{1}^{3}+4900 x_{1}^{2}+7031500 x_{1}+2401000000,
$$

and the morphism $C \rightarrow E$ by

$$
x_{1}=-\frac{882,000(x-14)}{x^{3}+420 x-5600}, \quad y_{1}=\frac{49,000 y\left(x^{3}-21 x^{2}-140\right)}{\left(x^{3}+420 x-5600\right)^{2}} .
$$


(Note that the $j$-invariant of $E$ is $-2^{5} \cdot 7 \cdot 17^{3}$, which matches the expression given for $j_{1}$ at $s=5, m=13$.)

\subsubsection{Further remarks}

The above calculation produced the morphism $C \rightarrow E_{1}$ to one of the associated elliptic curves, for a single point on the moduli space. To produce it over the whole base, one may work with the tautological genus 2 curve, and with coefficients in the function field of $\widetilde{\mathcal{L}}_{3}$. Alternatively, we may sample many such points and interpolate to find the coefficients of the rational map defining the morphism, and also the Weierstrass coefficients of the elliptic curve. Once the map is produced, it is trivial to verify it (by substituting in to the equation of the elliptic curve). To find the other map $C \rightarrow E_{2}$, we may use the other normalized differential on the genus 2 curve and apply the above process to it. More simply, we may rewrite the equation of $E_{1}$ and the map $C \rightarrow E_{1}$ in terms of the coordinates $r, s$ on the Humbert surface and the square root $z$ defining the double cover, and conjugate $z$ to $-z$ everywhere.

We omit the details of these calculations for the higher discriminants treated in this paper, as the expressions become much more complicated with increasing discriminant. We may, in fact, use the knowledge of the $j$-invariants obtained by the first (sampling and interpolation) method to reduce our work in this process, since they give us an extra equation satisfied by the Weierstrass coefficients $F, G, H$.

Finally, the above process hinges on finding normalized Weierstrass forms for the genus 2 curves in the first place. For a single point $\left(r_{0}, s_{0}, z_{0}\right)$ on the moduli space (with $r_{0}, s_{0} \in \mathbb{Q}$ say), i.e. for a single genus 2 curve, we do this by applying the eigenform location algorithm of [20], which indicates the fractional linear transformation needed to transform the curve to normalized form. At that point, the Weierstrass coefficients are floating point numbers, but we may apply a version of rational reconstruction to recognize them as elements of $\mathbb{Q}\left(z_{0}\right)$. Finally, with enough sampling, we may interpolate to write a normalized tautological curve over the moduli space.

\subsection{Comparison with previous results}

Comparing our absolute invariants $I_{4} / I_{2}^{2}, I_{2} I_{4} / I_{6}, I_{4} I_{6} / I_{10}$ to those of Shaska, we obtain the relation between the coordinates here and those in [26]. It is given by

$$
r=4096 r_{2}^{3} / r_{1}^{4}, \quad s=-48 r_{2} / r_{1},
$$

with inverse

$$
r_{1}=-s^{3} /(27 r), \quad r_{2}=s^{4} /(1296 r) .
$$

Using this change of coordinates, we see that the double cover is the same as the $(u, v)$ double cover in [26]. ${ }^{4}$

Let us check the compatibility of the $j$-invariants we found above with the values in [26]. In the setup of that paper, the genus 2 curve is given by

$$
y^{2}=\left(x^{3}+a x^{2}+b x+1\right)\left(4 x^{3}+b^{2} x^{2}+2 b x+1\right)
$$

\footnotetext{
${ }^{4}$ There is a typo in equation (14) of [26]: the numbers 27 and 1296 should be in the denominator rather than the numer-
} ator. 
where $a, b$ are related to the parameters $u, v$ on the Hilbert modular surface by

$$
u=a b, \quad v=b^{3} .
$$

Note that the parameters $r_{1}, r_{2}$ on the Humbert surface are

$$
\begin{aligned}
& r_{1}=\frac{v(v-2 u-9)^{3}}{27\left(4 v^{2}-u^{2} v-18 u v+27 v+4 u^{3}\right)}, \\
& r_{2}=-\frac{v(v-2 u-9)^{4}}{1296(v-27)\left(4 v^{2}-u^{2} v-18 u v+27 v+4 u^{3}\right)} .
\end{aligned}
$$

Define $^{5} R=4 a^{3}+27-18 a b-a^{2} b^{2}+4 b^{3}, F(x)=x^{3}+a x^{2}+b x+1$, and consider the rational functions $v=y\left(x^{3}-b x-2\right) / F(x)^{2}$ and $u=x^{2} /\left(x^{3}+a x^{2}+b x+1\right)$. Then $C$ maps to the genus 1 curve

$$
v^{2}=u^{3}+\frac{2\left(a b^{2}-6 a^{2}+9 b\right)}{R} u^{2}+\frac{12 a-b^{2}}{R} u-\frac{4}{R} .
$$

Computing the $j$-invariant of this elliptic curve, and using the transformation formulas

$$
u=(m s+27 s-m+9) / 4, \quad v=-(m-27) / 2,
$$

we see that it agrees with $j_{2}$.

\subsection{Special loci}

We now list several curves of interest on the Hilbert modular surface.

1. The rational curves $r=0, z= \pm(s-1)^{2}(2 s-1)$ correspond to $I_{10}=0$.

2. The curve $r=(s-1)^{2} / 4$ on the Humbert surface lifts to a rational curve, whose points correspond to Jacobians with endomorphisms by a split quaternion algebra. The associated elliptic curves are related by a 5 -isogeny.

3. The curves $s=5 / 9, z=4(19683 r-4) / 729$ are non-modular, but correspond to the "degenerate" case considered by Shaska in [26, equation(12)].

4. The modular genus 0 curve

$$
\begin{aligned}
& 4 s^{6}-20 s^{5}+41 s^{4}-432 r s^{3}-44 s^{3}-216 r s^{2}+26 s^{2}+576 r s \\
& -8 s+11664 r^{2}-184 r+1=0
\end{aligned}
$$

is part of the branch locus. It is parametrized by $(r, s)=\left((t+1)^{2}(t+4)^{4} /\left(729 t^{6}\right)\right.$, $\left.\left(11 t^{2}+24 t+16\right) /\left(6 t^{2}\right)\right)$ and corresponds to the two elliptic curves being isomorphic.

5. The curves $s=1, s=1 / 3$ lift to non-modular rational curves on $Y_{-}(9)$.

\section{Discriminant 16}

\subsection{Parametrization}

We start with a family of elliptic K3 surfaces with bad fibers of types $E_{7}$ and $D_{8}$, and a section of height $2=4-2$. A Weierstrass equation for such a K3 surface has the form

$$
y^{2}=x^{2}+t a(t) x^{2}+t^{4} b(t) x+t^{7} c
$$

\footnotetext{
${ }^{5}$ There is a typo on pg. 266 of [26]: in equation (6), the first factor in $\Delta$ should be the quantity $R$ as we have defined it, rather than its square.
} 
with $a, b$ linear in $t$ and $c$ constant. Since $a(0) \neq 0$ to have a fiber of type no worse than $D_{8}$ at 0 , we may write

$$
a=a_{0}\left(1+a_{1} t\right), \quad b=a_{0}\left(b_{0}+b_{1} t\right), \quad c=c_{0} a_{0} .
$$

We have a degree of freedom (scaling $t$ ), so we scale to have $a_{1}=1$. To have the leaves of the $D_{8}$ fiber defined over the ground field, we set $b_{0}=h+k$ and $c_{0}=h k$. Next, we impose a section of height 2: its $x$-coordinate must have the form $t^{3}\left(-h+g^{2} t\right)$ for some $g, h$. Substituting in to the Weierstrass equation and completing the square, we get a system of equations, which can be solve for $b_{1}$ and $k$. We finally use the remaining degree of freedom (Weierstrass scaling of $x$ and $y$ ) to set $g=1$, since the variable $g$ has weight 1 . Thus, we get a rational moduli space in the parameters $a_{0}$ and $h$. The Weierstrass equation suggests the convenient change of variables $h=2 s, a_{0}=2 r+h^{2} / 4$.

The final Weierstrass equation is

$$
y^{2}=x^{3}+\left(s^{2}+2 r\right) t(t+1) x^{2}-t^{4}\left(\left(s^{2}-2 r s+2 r\right) t-\left(2 s^{3}+4 r s+r^{2}\right)\right) x+2 r^{2} s t^{7} .
$$

Note that the section has $x$-coordinate $-2 s t^{3}+t^{4}$.

\subsection{Map to $\mathcal{A}_{2}$ and equation of $\tilde{\mathcal{L}}_{4}$}

We go to an $E_{8} E_{7}$ fibration by a 2-neighbor step, as illustrated below.

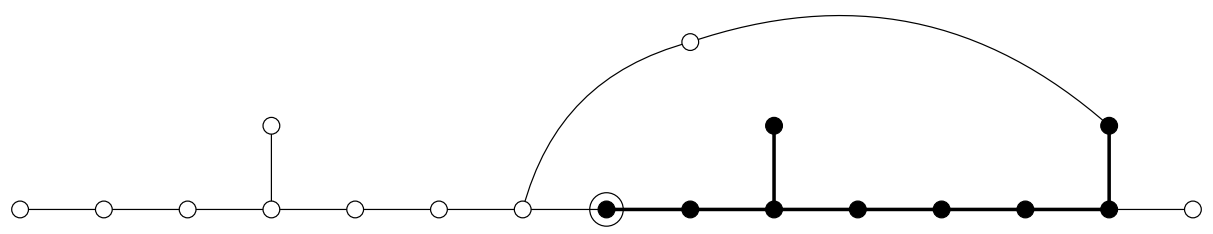

An elliptic parameter is $w=\left(x+r^{2} t^{3} /\left(s^{2}+2 r\right)\right) / t^{4}$. As in the previous section, we transform to the new elliptic fibration, which has the desired $E_{8}$ and $E_{7}$ fibers, and proceed to read out the Igusa-Clebsch invariants. They are given as follows:

$$
\begin{aligned}
I_{2}= & 2\left(3 s^{6}-12 r s^{5}+13 r^{2} s^{4}+18 r s^{4}-2 r^{3} s^{3}-48 r^{2} s^{3}+28 r^{3} s^{2}\right. \\
& \left.+36 r^{2} s^{2}+2 r^{4} s-48 r^{3} s+4 r^{4}+24 r^{3}\right) /\left(r^{2}\left(s^{2}-2 r s+2 r\right)\right), \\
I_{4}= & \left(s^{4}+24 s^{3}+4 r s^{2}+48 r s+r^{2}\right) / 4, \\
I_{6}= & \left(8 s^{10}-32 r s^{9}+192 s^{9}+34 r^{2} s^{8}-688 r s^{8}-4 r^{3} s^{7}+624 r^{2} s^{7}+1536 r s^{7}\right. \\
& -16 r^{3} s^{6}-4294 r^{2} s^{6}-8 r^{4} s^{5}+3000 r^{3} s^{5}+4608 r^{2} s^{5}-109 r^{4} s^{4} \\
& -8612 r^{3} s^{4}+26 r^{5} s^{3}+3488 r^{4} s^{3}+6144 r^{3} s^{3}+124 r^{5} s^{2}-5576 r^{4} s^{2} \\
& \left.+4 r^{6} s+480 r^{5} s+3072 r^{4} s+12 r^{6}+208 r^{5}\right) /\left(16 r^{2}\left(s^{2}-2 r s+2 r\right)\right), \\
I_{10}= & r^{2}\left(s^{2}+2 r\right)\left(s^{2}-2 r s+2 r\right) / 256 .
\end{aligned}
$$

We then compute the double cover defining the Hilbert modular surface.

Theorem 13 A birational model for the surface $\widetilde{\mathcal{L}}_{4}$ (equivalently, for $\left.Y_{-}(16)\right)$ is given by

$$
\begin{aligned}
z^{2}= & 2 s\left(s^{2}-2 r s+2 r\right) \\
& \times\left(2 s^{5}-4 r s^{4}-27 s^{4}+76 r s^{3}-32 r^{2} s^{2}-108 r s^{2}+144 r^{2} s-64 r^{3}-108 r^{2}\right) .
\end{aligned}
$$


It is a rational surface. The Humbert surface is birational to the $(r, s)$-plane. In these coordinates, the Igusa-Clebsch invariants of a point on the moduli space are given by the formulas in (4) above.

The right hand side is quartic in $r$, and we easily calculate that the surface is a rational elliptic surface with section. To find a rational parametrization, we make some elementary transformations to reduce the degree of the equation in $r$ and $s$. The net effect of these is to set

$$
r=\frac{-1}{e(e+2)^{2} f(2 e f-1)}, \quad s=\frac{1}{e(e+2) f}, \quad z=\frac{w}{e^{3}(e+2)^{5} f^{4}(2 e f-1)^{2}}
$$

The double cover is transformed to

$$
w^{2}=\left(108 e^{3}+160 e^{2}-144 e\right) f^{2}+\left(-54 e^{2}-88 e+8\right) f+4,
$$

which is a conic bundle over the $e$-line with an obvious section $(f, w)=(0,2)$. Parametrizing with a new parameter $d$, we obtain finally

$$
\begin{aligned}
r=- & \left(108 e^{3}+160 e^{2}-144 e-d^{2}\right)^{2} \\
& \quad /\left(2 e(e+2)^{2}\left(16 e^{2}+8 d e+128 e+d^{2}\right)\left(27 e^{2}+44 e+2 d-4\right)\right), \\
s= & \left(108 e^{3}+160 e^{2}-144 e-d^{2}\right) /\left(2 e(e+2)\left(27 e^{2}+44 e+2 d-4\right)\right) .
\end{aligned}
$$

where we have omitted $z$ for simplicity.

\subsection{Tautological curve}

Once again, it is possible to write down the tautological family of genus 2 curves over the Hilbert modular surface. However, the expression is quite long, and so we will omit it in the main body of the paper. However, it can be obtained from the auxiliary computer files. Similarly, for the higher discriminants treated in this paper, we will omit the expression for the tautological family of genus 2 curves (these are also in the computer files).

\subsection{Elliptic curves}

As before, it is possible to compute the equations of the two elliptic curves attached to the $(4,4)$-decomposable Jacobian, and the morphisms from the tautological genus 2 curve to the elliptic curves. We omit this calculation, and simply display the elementary symmetric functions in the $j$-invariants of the two elliptic curves.

$$
\begin{aligned}
j_{1}+j_{2}= & 128\left(4 s^{11}-8 r s^{10}-63 s^{10}+200 r s^{9}+184 s^{9}-128 r^{2} s^{8}-1144 r s^{8}\right. \\
& +1792 r^{2} s^{7}+1392 r s^{7}-768 r^{3} s^{6}-5372 r^{2} s^{6}+5888 r^{3} s^{5} \\
& +3936 r^{2} s^{5}-2048 r^{4} s^{4}-9840 r^{3} s^{4}+7168 r^{4} s^{3}+4928 r^{3} s^{3} \\
& \left.-2048 r^{5} s^{2}-6464 r^{4} s^{2}+2048 r^{5} s+2304 r^{4} s-256 r^{5}\right) /\left(s^{2}+2 r\right)^{4}, \\
j_{1} j_{2}= & 4096\left(s^{4}+24 s^{3}-56 r s^{2}+48 r s+16 r^{2}\right)^{3} /\left(s^{2}+2 r\right)^{4} .
\end{aligned}
$$

Over the Hilbert modular surface, which is rational in parameters $d, e$, we can solve the quadratic equation to get the two $j$-invariants. 


$$
\begin{aligned}
j_{1}= & 16\left(1080 e^{3}+108 d e^{2}-976 e^{2}-32 d e-96 e-d^{2}\right)^{3} \\
& /\left(e^{2}\left(16 e^{2}+8 d e+128 e+d^{2}\right)\left(27 e^{2}+44 e+2 d-4\right)^{3}\right), \\
j_{2}= & 16\left(24 e^{3}-12 d e^{2}-16 e^{2}-16 d e-96 e-d^{2}\right)^{3} \\
& /\left(e^{2}(e+2)^{4}\left(16 e^{2}+8 d e+128 e+d^{2}\right)\left(27 e^{2}+44 e+2 d-4\right)\right) .
\end{aligned}
$$

\subsection{Comparison with previous work}

We compare our formulas with those of Bolza [15, pp. 477,480], since those of Bruin and Doerksen are equivalent [2, Appendix A]. Bolza gives a family of genus 2 curves with $(4,4)$-reducible Jacobians over a weighted projective space with $\lambda, \mu, v$ of weights $1,2,3$ respectively. By comparing the Igusa-Clebsch invariants, we obtain the following relation between those coordinates and ours:

$$
\frac{\mu}{\lambda^{2}}=\frac{(4 e+d+16)(12 e+3 d-16)}{3\left(16 e^{2}+8 d e+128 e+d^{2}\right)}, \quad \frac{v}{\lambda^{3}}=\frac{(4 e+d+16)^{2}}{\left(16 e^{2}+8 d e+128 e+d^{2}\right)} .
$$

with inverse

$$
d=\frac{8\left(7 v^{2}+18 \lambda \mu \nu-16 \lambda^{3} v-9 \lambda^{2} \mu^{2}\right)}{9(v-\lambda \mu)^{2}}, \quad e=\frac{-2\left(v^{2}+6 \lambda \mu \nu-16 \lambda^{3} v+9 \lambda^{2} \mu^{2}\right)}{9(v-\lambda \mu)^{2}} .
$$

\subsection{Special loci}

1. The curves $r=0, z=4 s^{2}-54 s$ and $r=s^{2} /(2 s-2), z=0$ belong to the product locus. The latter is also part of the branch locus for the double cover $\widetilde{\mathcal{L}}_{4} \rightarrow \mathcal{L}_{4}$.

2. The curve $2 s^{3}+4 r s-r^{2}=0$ on $\mathcal{L}$ has genus 0 , and is parametrized by $(r, s)=\left(t^{2}(t-4) / 2, t(t-4) / 2\right)$. Its lift is also a rational curve, parametrized by letting $t=-\left(v^{2}+171\right) /(4(v-5))$. It is a modular curve, corresponding to the elliptic curves being 7 -isogenous.

3. The curve $s=0$ is part of the branch locus.

4. The curve $s^{5}-r s^{4}-s^{4}+2 r s^{3}-4 r s^{2}-4 r^{2}=0$ on the Humbert surface has genus 0 , and is parametrized by $(r, s)=\left(\left(t^{2}+2 t-2\right)^{2} /\left(t^{4}(t-1)\right),\left(t^{2}+2 t-2\right) /\right.$ $(t(t-1)))$. Its lift is also rational, parametrized by letting $t=u(1-2 u) /\left(u^{2}+2\right)$. This modular curve corresponds to the elliptic curves being 3-isogenous.

5. The curve $2 s^{5}-4 r s^{4}-27 s^{4}+76 r s^{3}-32 r^{2} s^{2}-108 r s^{2}+144 r^{2} s-64 r^{3}-108 r^{2}=0$ is part of the branch locus. It has genus 0 , and is parametrized by

$$
(r, s)=\left(\frac{27(t-1)\left(3 t^{2}+10 t-1\right)^{2}}{8(3 t-1)^{4}}, \frac{9(t+1)\left(3 t^{2}+10 t-1\right)}{(3 t-1)^{2}}\right) .
$$

6. There are also several simple non-modular curves: for instance, the specializations $s=9$ and $s=63 / 8$ gives genus 0 curves with rational points.

\section{Discriminant 25}

\subsection{Parametrization}

Next, we go on to discriminant 25, which was studied in [23]. We start with a family of elliptic K3 surfaces with bad fibers of type $D_{9}$ and $A_{6}$, and a section of height $25 / 28=4-9 / 4-6 / 7$. A Weierstrass equation for such a K3 surface has the form 


$$
y^{2}=x^{3}+\left(a_{0}+a_{1} t+a_{2} t^{2}+a_{3} t^{3}\right) x^{2}+2 t\left(b_{0}+b_{1} t+b_{2} t^{2}\right) x+\left(c_{0}+c_{1} t\right)^{2} t^{2} .
$$

This has a $D_{9}$ fiber at $t=\infty$ and an $A_{1}$ fiber at $t=0$. We have shifted $x$ so that the generator of the Mordell-Weil group has $x$-coordinate 0 . We need five more orders of vanishing for the discriminant at $t=0$. Since the components of the $A_{6}$ fiber are defined over the ground field, $a_{0}$ is a square; we may normalize it to be 1 . This takes care of the scaling in $x$. We then proceed to successively set the coefficients of $t^{2}$ through $t^{5}$ in the expression for the discriminant to be zero, which gives us the conditions

$$
\begin{aligned}
& c_{0}=b_{0}, \\
& c_{1}=\left(2 b_{1}+b_{0}^{2}-a_{1} b_{0}\right) / 2, \\
& b_{2}=-\left(\left(8 b_{0}-4 a_{1}\right) b_{1}+8 b_{0}^{3}-10 a_{1} b_{0}^{2}+\left(3 a_{1}^{2}-4 a_{2}\right) b_{0}\right) / 8, \\
& a_{3}=\left(2 b_{1}+3 b_{0}^{2}-2 a_{1} b_{0}\right)\left(4 b_{1}+10 b_{0}^{2}-8 a_{1} b_{0}-4 a_{2}+a_{1}^{2}\right) /\left(8 b_{0}\right) .
\end{aligned}
$$

To simplify the succeeding expressions, we define a new variable $k$ by $b_{1}=k+a_{1} b_{0} / 2$. The condition for the final order of vanishing gives us a quadratic equation in $a_{2}$, whose discriminant is a square times $4 k^{2}-5 b_{0}^{4}+2 a_{1} b_{0}^{3}$. Therefore, setting

$$
a_{1}=\left(h^{2}-4 k^{2}+5 b_{0}^{4}\right) /\left(2 b_{0}^{3}\right)
$$

makes the expression a square, and we solve for $a_{2}$, obtaining

$$
\begin{aligned}
a_{2}= & \left(16 k^{4}-32 b_{0}^{2} k^{3}-8 h^{2} k^{2}+16 b_{0}^{2} h k^{2}-24 b_{0}^{4} k^{2}+8 b_{0}^{2} h^{2} k\right. \\
& \left.+16 b_{0}^{4} h k+8 b_{0}^{6} k+h^{4}-4 b_{0}^{2} h^{3}-2 b_{0}^{4} h^{2}+4 b_{0}^{6} h+5 b_{0}^{8}\right) /\left(16 b_{0}^{6}\right) .
\end{aligned}
$$

We also scale $b_{0}=1$ to quotient by the scaling in $t$, and let

$$
h=s-r, \quad k=(r+s-1) / 2
$$

to simplify the expressions; this change of variable is suggested by the final formulas below. We obtain the final Weierstrass equation

$$
\begin{aligned}
y^{2}= & x^{3}+\left(r^{2}(2 r-1) s^{2} t^{3}+\left(4 r^{2} s^{2}-4 r s^{2}+s^{2}-12 r^{2} s+10 r s+r^{2}\right) t^{2} / 4\right. \\
& -(2 r s-s-r-2) t+1) x^{2}-t\left(\left(2 r s^{2}-s^{2}+6 r^{2} s-6 r s-r^{2}\right) t^{2} / 2\right. \\
& +(2 r s-2 s-2 r-1) t-2) x+t^{2}(s t+r t+2)^{2} / 4 .
\end{aligned}
$$

As noted above, the section of height 25 / 28 has $x$-coordinate 0.

\subsection{Map to $\mathcal{A}_{2}$ and equation of $\tilde{\mathcal{L}}_{5}$}

We then proceed to an elliptic fibration with $E_{8}$ and $A_{7}$ fibers, via a 2-neighbor step. The elliptic parameter is simply $x$. 


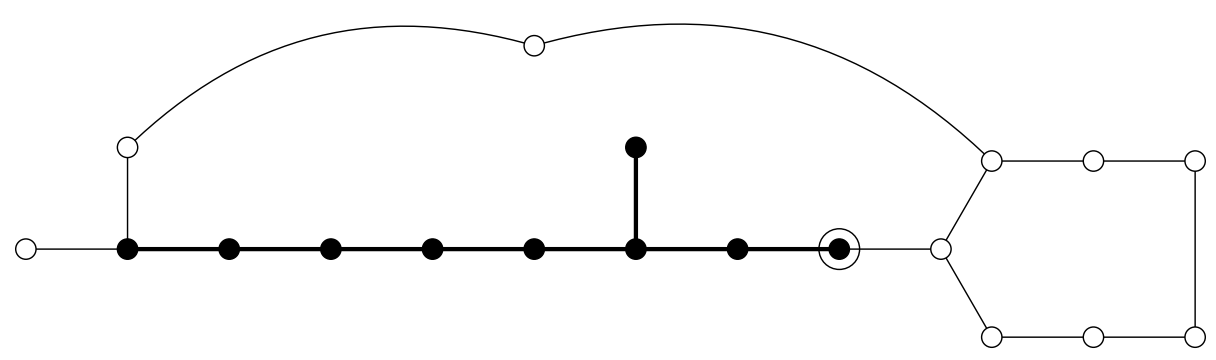

After some easy Weierstrass transformations and a fractional linear transformation of the elliptic parameter, the Weierstrass equation for the new fibration is

$$
\begin{aligned}
y^{2}= & x^{3}+\left(12 r^{3} s t^{3}+\left(4 r^{2} s^{2}-4 r s^{2}+s^{2}+12 r^{2} s-14 r s+r^{2}\right) t^{2}\right. \\
& -4(2 r s+s+r-1) t+4) x^{2} / 16 \\
& +t^{4}\left(6 r^{6} s^{2} t^{2}+r^{3} s\left(4 r^{2} s^{2}-4 r s^{2}+s^{2}+12 r^{2} s-14 r s+r^{2}\right) t\right. \\
& \left.-2 r s\left(4 r^{2} s^{2}-4 r s^{2}+s^{2}+2 r^{3} s+r^{2} s+r^{3}-r^{2}\right)\right) x / 32 \\
& +t^{8}\left(4 r^{9} s^{3} t+r^{6} s^{2}\left(4 r^{2} s^{2}-4 r s^{2}+s^{2}+12 r^{2} s-14 r s+r^{2}\right)\right) / 256 .
\end{aligned}
$$

There is a section $P$ of height $25 / 8=4-7 / 8$. Finally, we take a 2 -neighbor step to an elliptic fibration with $E_{8}$ and $E_{7}$ fibers.

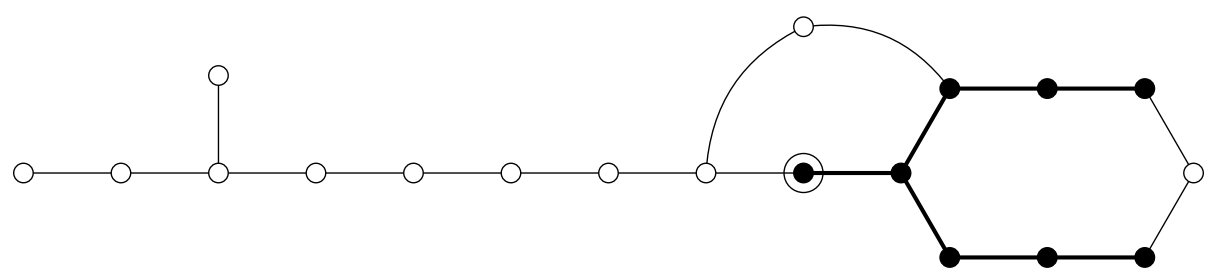

The elliptic parameter is $x / t^{4}$. If $F$ is the class of the $E_{7}$ fiber, then $P \cdot F=3$, while $F \cdot C=2$, where $C$ is the remaining component of the $A_{7}$ fiber not includes in $F$. Since these numbers are coprime, the genus 1 fibration defined by $F$ has a section. We therefore convert to the Jacobian, and read out the Igusa-Clebsch invariants, which are as follows.

$$
\begin{aligned}
I_{2}=2 & \left(4 r^{2} s^{2}+20 r s^{2}+s^{2}-12 r^{2} s+22 r s-6 s+r^{2}-6 r+3\right), \\
I_{4}=( & 16 r^{4} s^{4}-32 r^{3} s^{4}+24 r^{2} s^{4}-8 r s^{4}+s^{4}+96 r^{4} s^{3}-976 r^{3} s^{3}+904 r^{2} s^{3} \\
& \left.-220 r s^{3}+56 r^{4} s^{2}-392 r^{3} s^{2}+198 r^{2} s^{2}-24 r^{4} s+20 r^{3} s+r^{4}\right) / 4, \\
I_{6}=\left(64 r^{6} s^{6}+320 r^{5} s^{6}-784 r^{4} s^{6}+608 r^{3} s^{6}-196 r^{2} s^{6}+20 r s^{6}+s^{6}+64 r^{6} s^{5}\right. & -4128 r^{5} s^{5}-17120 r^{4} s^{5}+18544 r^{3} s^{5}-3692 r^{2} s^{5}-378 r s^{5}-8 s^{5} \\
& -1616 r^{6} s^{4}+10304 r^{5} s^{4}-35208 r^{4} s^{4}+30368 r^{3} s^{4}-10769 r^{2} s^{4} \\
& +1720 r s^{4}+4 s^{4}-896 r^{6} s^{3}+6192 r^{5} s^{3}-7152 r^{4} s^{3}-1732 r^{3} s^{3} \\
& +3792 r^{2} s^{3}-880 r s^{3}+364 r^{6} s^{2}-2228 r^{5} s^{2}+4543 r^{4} s^{2}-3312 r^{3} s^{2} \\
& \left.+792 r^{2} s^{2}-36 r^{6} s+238 r^{5} s-264 r^{4} s+80 r^{3} s+r^{6}-8 r^{5}+4 r^{4}\right) / 8, \\
I_{10}=- & 16 r^{7}(2 r-1)^{2} s^{5} .
\end{aligned}
$$

Computing the double cover, we obtain the following description of the moduli space. 
Theorem 14 A birational model for the surface $\widetilde{\mathcal{L}}_{5}$ (equivalently, for $Y_{-}(25)$ ) is given by

$$
z^{2}=\left(4 s^{2}+22 s-1\right)^{2} r^{2}-2 s\left(8 s^{3}-88 s^{2}+72 s-17\right) r+s^{2}(2 s-1)^{2} .
$$

It is a rational surface. The Humbert surface is birational to the (r, s)-plane. In these coordinates, the Igusa-Clebsch invariants of a point on the moduli space are given by the formulas in (5) above.

Once again, this is a conic bundle with a section. Setting $z=r m+s(2 s-1)$ and solving for $r$, we obtain

$$
r=-\frac{2 s\left(2 s m-m+8 s^{3}-88 s^{2}+72 s-17\right)}{\left(m-4 s^{2}-22 s+1\right)\left(m+4 s^{2}+22 s-1\right)} .
$$

\subsection{Comparison with previous work}

Let us compare our results with those of [23]. There it is asserted that the moduli space $\mathcal{L}_{5}$ of genus 2 curves with a degree 5 elliptic subfield (which is birational to the Humbert surface $\mathcal{H}_{25}$ ), is birational to the hypersurface cut out by the following equation in $\mathbb{A}_{u, v, w}^{3}$ :

$$
\begin{aligned}
0=64 & v^{2}(u-4 v+1)^{2} w^{2}+4 v\left(128 v^{4}-16 u^{2} v^{3}+288 u v^{3}\right. \\
& -2592 v^{3}-24 u^{3} v^{2}+96 u^{2} v^{2}+272 u v^{2}+4672 v^{2} \\
& \left.+15 u^{4} v-92 u^{3} v+20 u^{2} v-576 u v-2 u^{5}+12 u^{4}-4 u^{3}\right) w \\
& +\left(u^{2}+4 v u+4 v^{2}-48 v\right)^{3} .
\end{aligned}
$$

In fact, this is a rational surface: the discriminant with respect to $w$ is

$$
-(v+2 u-16)\left(16 v^{2}-u^{2} v+36 u v+108 v-2 u^{3}\right)
$$

and the invertible change of variables

$$
u=\left(v_{1}+25\right) h-18, \quad v=-2\left(v_{1}+25\right) h+\left(v_{1}+52\right)
$$

converts it to $\left(h^{2} v_{1}+24 h^{2}-8 h-16\right) / v_{1}$, up to squares. Setting this quantity equal to $g^{2}$ and solving for $v_{1}$, we obtain the full parametrization

$$
\begin{aligned}
u & =\left(h^{3}-10 h^{2}-25 g^{2} h+16 h+18 g^{2}\right) /\left(h^{2}-g^{2}\right) \\
v & =-2\left(h^{3}-6 h^{2}-25 g^{2} h+12 h+26 g^{2}-8\right) /\left(h^{2}-g^{2}\right) \\
w & =\frac{(h-5 g+4)(h+5 g+4)^{2}\left(3 h^{2}-15 g h-10 h+14 g+8\right)^{3}}{16(h-1)(h-g)^{2}(3 h-15 g-8)^{2}\left(h^{3}-6 h^{2}-25 g^{2} h+12 h+26 g^{2}-8\right)} .
\end{aligned}
$$

Remark 15 Unfortunately, there is a mistake ${ }^{6}$ in the paper [23]: the moduli space described there, claimed to be birational to $\mathcal{L}_{5}$ in Theorem 3 of their paper, is actually birational to the Hilbert modular surface (i.e. to $\widetilde{\mathcal{L}}_{5}$ ), and the Humbert surface is a quotient of it by an involution which we give explicitly in the auxiliary files (we check directly that the absolute Igusa-Clebsch invariants are not changed by applying this involution).

${ }^{6}$ We take this opportunity to point out another typo: the last term for $a_{0}$ in equation (10) should be $12 z a$ rather than $12 y a$. 
By comparing the Igusa-Clebsch invariants, we obtain the relation between these coordinates and ours:

$$
\begin{aligned}
s=( & +g)(3 h-15 g-8) /\left(4\left(h^{2}-3 g h-10 g^{2}-2 g+4\right)\right), \\
m=( & 73 h^{5}-571 g h^{4}-208 h^{4}+310 g^{2} h^{3}+644 g h^{3}+312 h^{3}+3350 g^{3} h^{2} \\
& +2220 g^{2} h^{2}-856 g h^{2}-768 h^{2}+625 g^{4} h \\
& -1700 g^{3} h-2120 g^{2} h+64 g h+192 h+3125 g^{5} \\
& \left.+2500 g^{4}-5400 g^{3}-4160 g^{2}+1344 g+1024\right) \\
& /\left(4(h-g)\left(h^{2}-3 g h-10 g^{2}-2 g+4\right)^{2}\right)
\end{aligned}
$$

with inverse

$$
\begin{aligned}
& g=\frac{s m^{2}+8 s^{3} m-68 s^{2} m+2 s m+8 m+16 s^{5}-272 s^{4}+1228 s^{3}-212 s^{2}-127 s-8}{\left(m+4 s^{2}-28 s-1\right)\left(2 s m-m+8 s^{3}-88 s^{2}+72 s-17\right)}, \\
& h=\frac{s m^{2}+8 s^{3} m-124 s^{2} m+74 s m-8 m+16 s^{5}-496 s^{4}+3484 s^{3}-3036 s^{2}+649 s+8}{\left(m+4 s^{2}-28 s-1\right)\left(2 s m-m+8 s^{3}-88 s^{2}+72 s-17\right)} .
\end{aligned}
$$

\subsection{Elliptic curves}

The $j$-invariants of the associated elliptic curves are given by

$$
\begin{aligned}
j_{1}=- & \left(m^{4}+16 s^{2} m^{3}-8 s m^{3}+20 m^{3}+96 s^{4} m^{2}-8288 s^{3} m^{2}+10632 s^{2} m^{2}\right. \\
& -5304 s m^{2}+1014 m^{2}+256 s^{6} m-65920 s^{5} m+116864 s^{4} m-104416 s^{3} m \\
& +49824 s^{2} m-24792 s m+5684 m+256 s^{8}-131584 s^{7}+4492928 s^{6} \\
& \left.-5084160 s^{5}+1924400 s^{4}-243456 s^{3}-87256 s^{2}+14552 s+4945\right)^{3} \\
& /\left(4\left(m+4 s^{2}+22 s-1\right)^{5}\left(2 s m-m+8 s^{3}-88 s^{2}+72 s-17\right)^{3}\right. \\
& \left.\left(m^{2}+8 s^{2} m-4 s m+16 s^{4}-528 s^{3}-188 s^{2}-24 s-1\right)\right), \\
j_{2}=- & \left(m^{4}+16 s^{2} m^{3}-88 s m^{3}+20 m^{3}+96 s^{4} m^{2}-1568 s^{3} m^{2}+2632 s^{2} m^{2}\right. \\
& -1064 s m^{2}+54 m^{2}+256 s^{6} m-8320 s^{5} m+57984 s^{4} m-33696 s^{3} m \\
& +6944 s^{2} m+6328 s m-1996 m+256 s^{8}-13824 s^{7}+207488 s^{6}-916480 s^{5} \\
& \left.+510000 s^{4}-115456 s^{3}-53016 s^{2}+19592 s+2065\right)^{3} \\
& /\left(8\left(m-4 s^{2}-22 s+1\right)^{5}\left(2 s m-m+8 s^{3}-88 s^{2}+72 s-17\right)^{2}\right. \\
& \left.\left(m^{2}+8 s^{2} m-4 s m+16 s^{4}-528 s^{3}-188 s^{2}-24 s-1\right)\right) .
\end{aligned}
$$

Let us check that the $j$-invariant agrees with that implicit in [23]. It follows from the description there that $C$ maps to the elliptic curve

$$
y^{2}=x(x-1)(x-\lambda)
$$

where $\lambda=\zeta F_{1}(\zeta)^{2} / F_{2}(\zeta)^{2}$ where $F_{1}$ and $F_{2}$ are the polynomials

$$
\begin{aligned}
& F_{1}(T)=T^{2}+\left(2 a+2 b+a^{2}\right) T+2 a b+b^{2} \\
& F_{2}(T)=(2 a+1) T^{2}+\left(a^{2}+2 a b+2 b\right) T+b^{2},
\end{aligned}
$$

$\zeta$ is a root of

$$
(2 a+1) \zeta^{2}+\left(2 b-2 a b-2 a-a^{2}\right) \zeta+b^{2}+2 a b=0
$$


and $u, v$ are related to the parameters $a, b$ by

$$
u=\frac{2 a\left(a b+b^{2}+b+a+1\right)}{b(a+b+1)}, \quad v=\frac{a^{3}}{b(a+b+1)} .
$$

After some computation, the value of the $j$-invariant is found to agree with $j_{1}$.

\subsection{Special loci}

We again list several interesting curves on the surface.

1. The curves $r=0, z= \pm s(2 s-1), s=0, z= \pm r$ are rational curves corresponding to $I_{10}=0$, as is the lift of $r=1 / 2$.

2. The curve $4 r^{2} s^{2}-4 r s^{2}+s^{2}+6 r^{2} s-2 r s-2 r^{3}+r^{2}=0$ on the Humbert surface, parametrized by $(r, s)=\left(\left(t^{2}-2 t+4\right) / 8,(t-2)\left(t^{2}-2 t+4\right) /\left(4 t^{2}\right)\right)$, lifts to an elliptic curve on $Y_{-}$(25). It parametrizes the locus for which the two associated elliptic curves are related by a 14-isogeny. Abstractly, this elliptic curve is isomorphic to $y^{2}+x y+y=x^{3}+4 x-6$ of conductor 14 , which has rank 0 .

3. The curve $(2 r-1)^{2} s+r=0$ lifts to an elliptic curve on $Y_{-}$(25), isomorphic to the elliptic curve $y^{2}+y=x^{3}-x^{2}-10 x-20$ of conductor 11 and rank 0 . It parametrizes the locus of reducible Jacobians for which the two associated elliptic curves have an 11-isogeny.

4. The curve

$$
\begin{aligned}
& 4(2 r-1)^{2} s^{4}+4\left(44 r^{2}+44 r-1\right) s^{3}+\left(476 r^{2}-144 r+1\right) s^{2} \\
& \quad+\left(-44 r^{2}+34 r\right) s+r^{2}=0
\end{aligned}
$$

of genus 0 is part of the branch locus, and is parametrized by $(r, s)=\left((t+1)^{2}\left(2 t^{2}+4 t+1\right) /\left(2\left(t^{2}+t-1\right)^{2}\right),-\left(2 t^{2}+4 t+1\right) /\left(2 t^{2}\right)\right)$.

It corresponds to the two elliptic curves being isomorphic.

5. The curve parametrized by

$$
r=\frac{1}{50 t}, \quad s=\frac{(9 t-1)(25 t-1)}{16\left(25 t^{2}+6 t+1\right)}, \quad m=\frac{25\left(37375 t^{4}+7044 t^{3}+762 t^{2}-124 t-1\right)}{64\left(25 t^{2}+6 t+1\right)^{2}}
$$

corresponds to the degenerate Case I of [23].

6. The curve parametrized by

$$
r=\frac{-3(25 t-1)}{2(25 t-16)}, \quad s=\frac{1-t}{2}, \quad m=\frac{15625 t^{3}+3750 t^{2}-7800 t+89}{(t-1)(25 t-16)^{2}}
$$

corresponds to the degenerate Case II of [23].

7. The curve parametrized by

$$
r=\frac{1}{2(t-7)}, \quad s=\frac{3}{8}, \quad m=-\frac{125}{16}
$$

corresponds to the degenerate case III of [23]. 


\section{Discriminant 36}

\subsection{Parametrization}

Next, we describe the moduli space for degree 6 covers. The corresponding Humbert surface is birational to the moduli space of elliptic K3 surfaces with $E_{8}, A_{5}$ and $A_{2}$ fibers, with a section of height $2=4-2 / 3-2 \cdot 4 / 6$. We briefly describe how to parametrize this moduli space and the family of K3 surfaces over it.

Start with an elliptic surface

$$
y^{2}=x^{3}+a x^{2}+2 b t^{2}(t-1) x+c t^{4}(t-1)^{2}
$$

where $a, b, c$ are polynomials of degrees $2,1,1$ respectively. This surface already has an $E_{8}$ fiber at $\infty$, an $A_{3}$ fiber at 0 and an $A_{1}$ fiber at $t=1$. Since the components of the eventual $A_{5}$ fiber at $t=0$ are rational, the constant term of $a$ is a square, and we may scale $x$ and $y$ so that $a(0)=1$. Similarly, $a(1)$ is rational. So we may write

$$
a=1-t+t e^{2}+a_{1} t(1-t) .
$$

Imposing two more orders of vanishing at $t=0$, we may solve linear equations for $c_{0}$ and $c_{1}$. We need one more order of vanishing of the discriminant at $t=1$. This gives a quadratic equation in $b_{1}$ whose discriminant is $-a_{1}$ times a square. We set $a_{1}=-f^{2}$ and solve for $b_{1}$. Finally, we need a section with the requisite height. It must have $x$-coordinate $t^{2}(t-1)$ times a linear factor, which we may normalize as $x_{0}+2 b_{0}+g^{2} t$. Substituting in to the Weierstrass cubic polynomial and completing the square, we can compute the $y$-coordinate. This leads to three equations involving the remaining variables. We eliminate $x_{0}$ and $b_{0}$ to get a single equation connecting the remaining variables $e, f, g$. This equation is cubic in $f$, but substituting $e=e^{\prime}-f$ and $g=g^{\prime} f$ makes it quadratic. Setting the discriminant equal to a square, after some easy algebra we end up with a parametrization by two variables $r$ and $s$. The universal Weierstrass equation is

$$
\begin{aligned}
y^{2}=x^{3} & +\left(9(r+6)^{2} s^{2}(2 s+r)^{2} t^{2} / 4-s\left(36 s-r^{2}\right)\left(18 r s^{2}+108 s^{2}+9 r^{2} s\right.\right. \\
& \left.\left.+36 r s+108 s+r^{3}+9 r^{2}+54 r\right) t / 3+(s-1)^{2}\left(36 s-r^{2}\right)^{2}\right) x^{2} \\
& +r(r+6)^{2} s^{3}(6 s+r) t^{2}(r t-12) \cdot\left(s \left(18 r s^{2}+108 s^{2}+9 r^{2} s\right.\right. \\
& \left.\left.+108 s+2 r^{3}+9 r^{2}+54 r\right) t-6(s-1)^{2}\left(36 s-r^{2}\right)\right) x \\
& +3 r^{2}(r+6)^{4} s^{6}(6 s+r)^{2} t^{4}(r t-12)^{2}\left(r s t+3 s^{2}-6 s+3\right) .
\end{aligned}
$$

Here we have introduced appropriate scalings of $x, y, t$ to eliminate denominators. The $x$-coordinate of the section of height 2 is given by

$$
x_{s}=3 r s^{3} t^{2}(r t-12)(3 s t-6 s-r+6) .
$$

\subsection{Map to $\mathcal{A}_{2}$ and equation of $\tilde{\mathcal{L}}_{6}$}

The 3-neighbor step corresponding to the figure below takes us to an $E_{8} E_{7}$ fibration. 


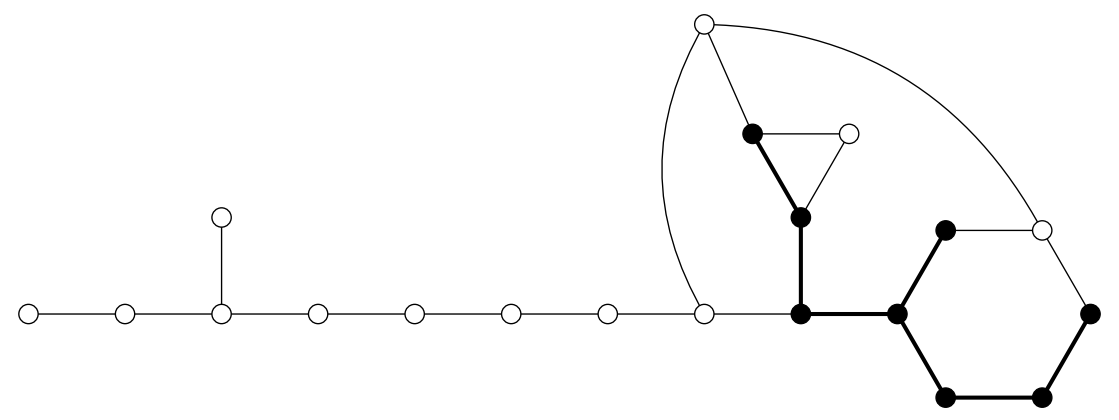

We may now read out the Igusa-Clebsch invariants. They are fairly lengthy to write down, so we will not display them here. Instead they may be accessed in the auxiliary computer files.

Theorem 16 A birational model for the surface $\widetilde{\mathcal{L}}_{6}$ (equivalently, for $Y_{-}(36)$ ) is given by

$$
\begin{aligned}
z^{2}= & \left(36 s-r^{2}\right)\left(1296(r+6)^{2} s^{3}-36 r(r+6)\left(r^{2}+162 r-648\right) s^{2}\right. \\
& \left.+12 r^{2}\left(11 r^{3}+207 r^{2}+2592 r+8748\right) s+r^{4}\left(7 r^{2}+108 r+972\right)\right) .
\end{aligned}
$$

It is a singular elliptic K3 surface (i.e. of maximal Picard number 20). The Humbert surface is birational to the $(r, s)$-plane.

The equation above shows that $\widetilde{\mathcal{L}}_{6}$ has a genus 1 fibration over $\mathbb{P}_{r}^{1}$, with an obvious section. Therefore it is an elliptic surface. Taking the corresponding Jacobian fibration and after some simple transformations (including setting $r=4 t$ ), we obtain the following Weierstrass equation, showing that it is a K3 surface.

$$
\begin{aligned}
y^{2}=x^{3} & +3\left(t^{2}-4 t-1\right)\left(3 t^{2}-4 t-3\right) x^{2} \\
& +96 t^{2}(t+1)(3 t+1)\left(t^{2}-6 t-3\right) x \\
& -256 t^{3}(3 t+1)^{2}\left(7 t^{3}-30 t^{2}-33 t-8\right) .
\end{aligned}
$$

It has reducible fibers of type $\mathrm{I}_{3}$ at $t=0$ and at the roots of $t^{3}-3 t^{2}-3 t-1, \mathrm{I}_{4}$ at $t=-1 / 3$ and at $t=\infty$, and $\mathrm{I}_{2}$ at the roots of $t^{2}-6 t-3$. The trivial lattice therefore has rank $2+3+2+6+3+2=18$, leaving room for Mordell-Weil rank up to 2 . We find the sections

$$
\begin{aligned}
& P=\left(8(3 t+1)\left(t^{2}-2 t-1\right), 8(3 t+1)^{2}\left(t^{3}-3 t^{2}-3 t-1\right)\right) \\
& Q=\left(-4 t(t-1)(3 t+1), 4 t(3 t+1)^{2}\left(t^{2}-6 t-3\right)\right) .
\end{aligned}
$$

The intersection matrix of these sections is

$$
\frac{1}{12}\left(\begin{array}{ll}
3 & 3 \\
3 & 7
\end{array}\right)
$$


It has determinant $1 / 12$. Hence the span of these two sections and the trivial lattice has rank 20 and discriminant $3^{4} \cdot 4^{2} \cdot 2^{2} \cdot(1 / 12)=2^{4} \cdot 3^{3}$. We check that it is 2 - and 3 - saturated in the Néron-Severi lattice, and therefore it must be all of NS(Y-(36)).

\subsection{Elliptic curves}

We compute the symmetric polynomials in the two $j$-invariants, in terms of the parameters on the Humbert surface, and check that the discriminant of the polynomial $T^{2}-\left(j_{1}+j_{2}\right) T+j_{1} j_{2}$ is, up to squares, the same as the branch locus for the Hilbert modular surface. We obtain the following expressions.

$$
\begin{aligned}
j_{1}+j_{2}= & -54\left(1119744(r+6)^{5} s^{9}-31104 r(r+6)^{4}\left(r^{2}+162 r-1944\right) s^{8}\right. \\
& +31104 r^{2}(r+6)^{3}\left(4 r^{3}+51 r^{2}-3888 r+43740\right) s^{7} \\
& -7776 r^{3}(r+6)^{2}\left(5 r^{4}-726 r^{3}+9540 r^{2}+36936 r-2099520\right) s^{6} \\
& -2592 r^{4}(r+6)\left(31 r^{5}-777 r^{4}-756 r^{3}+208008 r^{2}-5458752 r\right. \\
& -42515280) s^{5}-216 r^{5}\left(301 r^{6}+1002 r^{5}-32472 r^{4}-1228608 r^{3}\right. \\
& \left.-59731344 r^{2}-629226144 r-1836660096\right) s^{4}-216 r^{6}\left(50 r^{6}\right. \\
& -8601 r^{5}-256410 r^{4}-3320352 r^{3}-32122656 r^{2} \\
& -195570288 r-459165024) s^{3}-54 r^{8}(5 r+54)\left(143 r^{4}\right. \\
& \left.+4248 r^{3}+61776 r^{2}+419904 r+944784\right) s^{2} \\
& \left.+6 r^{11}(5 r+54)^{2}(11 r+162) s+r^{12}(5 r+54)^{3}\right) /\left(r^{11}(6 s+r)^{4}\right), \\
j_{1} j_{2}= & 729\left(144(r+6)^{2} s^{4}-288 r(r+6)(r+114) s^{3}+72 r^{2}\left(11 r^{2}+108 r+540\right) s^{2}\right. \\
& \left.+24 r^{3}(r+18)(5 r+54) s+r^{4}(5 r+54)^{2}\right)^{3} /\left(r^{12}(6 s+r)^{6}\right) .
\end{aligned}
$$

\subsection{Special loci}

We next present some curves of low genus on the modular surface.

1. The curves $r=0$ and $r=-6 s$ lift to pairs of rational curves on $Y_{-}$(36). They correspond to $I_{10}=0$.

2. The curve $s=r^{2} / 36$ lies in the branch locus. It is also a component of the product locus (i.e. the Jacobian degenerates to a product of elliptic curves).

3. The curve $r=-6$ also belongs to the product locus. It lifts to a genus 0 curve without any real points.

4. The curve $s=0$ lifts to a genus 0 curve whose points have $j_{1}=j_{2}$. It too does not have any real points.

5. The curve $s=1$ lifts to an elliptic curve isomorphic to $y^{2}+y=x^{3}-x^{2}-10 x-20$, of conductor 11 and rank 0 . Its points correspond to reducible Jacobians such that the elliptic curves are related by an 11-isogeny.

6. The curve $2 r s+12 s-r^{2}+2 r=0$ lifts to an elliptic curve isomorphic to $y^{2}=x^{3}+22 x^{2}+125 x$, which has conductor 20 and rank 0 . Along it, the elliptic curves are related by a 5 -isogeny.

7. The curve $12 s^{2}+6 r s+r^{2}+2 r=0$ lifts to an elliptic curve isomorphic to $y^{2}=x^{3}+30 x^{2}+289 x$, which has conductor 17 and rank 0 . It corresponds to elliptic curves linked by a 17 -isogeny. 
8. The curve

$$
\begin{gathered}
\left(1296(r+6)^{2} s^{3}-36 r(r+6)\left(r^{2}+162 r-648\right) s^{2}+12 r^{2}\left(11 r^{3}+207 r^{2}\right.\right. \\
\left.+2592 r+8748) s+r^{4}\left(7 r^{2}+108 r+972\right)\right)=0
\end{gathered}
$$

is part of the branch locus. It has genus 0 and can be parametrized as

$$
r=\frac{-6(t+2)(t-1)^{2}}{t\left(t^{2}+2 t-1\right)}, \quad s=\frac{\left(1+t+t^{2}\right)(t+2)^{2}(t-1)^{2}}{\left(t^{2}+t-1\right)\left(t^{2}+2 t-1\right) t^{2}}
$$

It corresponds to an isogeny of degree 1 , i.e. $j_{1}=j_{2}$.

9. The curve

$$
\begin{aligned}
& 46656(r+6) s^{4}-216 r^{2}\left(r^{2}+12 r+180\right) s^{3}+36 r^{3}\left(7 r^{2}+96 r-108\right) s^{2} \\
& +72 r^{5}(r+12) s+r^{6}(5 r+54)=0
\end{aligned}
$$

on the Humbert surface has genus 0 , and can be parametrized by

$$
r=\frac{6\left(3 t^{2}-3 t+1\right)^{2}}{t\left(t^{3}-5 t^{2}+4 t-1\right)}, \quad s=\frac{(3 t-1)\left(3 t^{2}-3 t+1\right)^{3}}{(t-1) t^{3}\left(t^{3}-5 t^{2}+4 t-1\right)}
$$

It lifts to an elliptic curve isomorphic to $y^{2}+y=x^{3}-7$, which has conductor 27 and rank 0 . The points on this curve correspond to the two elliptic curves being related by a 3-isogeny.

(10) The curve $r=-2 s$ on the Humbert surface is non-modular. It lifts to an elliptic curve isomorphic to $y^{2}=x^{3}-9 x+9$, which has conductor 324 and rank 1 .

(11) We can also produce non-modular curves of genus 0 through the sections $P$ and $Q$ and their linear combinations. For instance, the section $P$ gives the curve

$s=\frac{r^{2}\left(r^{2}+9 r+162\right)}{54(r-36)(r+6)}, \quad z=\frac{-r^{3}\left(r^{2}-108 r-972\right)\left(r^{3}-54 r^{2}-972 r-5832\right)}{27(r-36)^{2}(r+6)}$

while $P-Q$ gives the curve

$$
\begin{aligned}
& s=\frac{-r\left(7 r^{2}+108 r+1296\right)}{6\left(r^{2}-108 r+216\right)}, \\
& z=\frac{-2(r-36) r^{2}(r+6)(7 r+12)\left(r^{3}-54 r^{2}-972 r-5832\right)}{\left(r^{2}-108 r+216\right)^{2}} .
\end{aligned}
$$

The specializations of the tautological family along these genus 0 curves are oneparameter families of genus 2 curves with real multiplication by $\mathcal{O}_{36}$.

\section{Discriminant 49}

\subsection{Parametrization}

We start with the family of elliptic K3 surfaces having $E_{8}, A_{4}, A_{2}$ and $A_{1}$ fibers at $t=\infty, 0,1, h$ respectively, and a section of height $49 / 30=4-6 / 5-2 / 3-1 / 2$.

We start with a Weierstrass equation

$$
y^{2}=x^{3}+a x^{2}+2 t(t-1)(t-h) b x+c t^{2}(t-h)^{2}(t-1)^{2}
$$

with $a, b, c$ of degrees $2,1,1$ respectively. We have shifted $x$ so that the section has vanishing $x$-coordinate at $t=0,1, h$. 
This fibration already has an $E_{8}$ fiber at $\infty$ and $A_{1}$ fibers at $t=0,1, h$. Let us normalize

$$
\begin{aligned}
& a=(1-t)+e^{2} t+a_{2} t(1-t) \\
& b=b_{0}+b_{1} t \\
& c=c_{0}+c_{1} t
\end{aligned}
$$

where we have used that $a(0)$ and $a(1)$ must be squares in order for the components to be defined over the base field, and also used the Weierstrass scaling to fix $a(0)=1$.

Imposing two more orders of vanishing at $t=0$ gives linear equations for $c_{0}$ and $c_{1}$, which are easily solved. A third order of vanishing gives a quadratic equation for $b_{1}$, whose discriminant equals $b_{0}(h+1)-a_{2}$, up to squares. We set it equal to $f^{2}$ and solve for $a_{2}$ and then $b_{1}$. Next, we impose an extra order of vanishing at $t=1$, which gives a quadratic equation for $e$, with discriminant up to squares equal to $f^{2}-b_{0}$. We therefore set $b_{0}=f^{2}-g^{2}$ and solve for $e$. Next, scale $f=f^{\prime} g$ and $m=m^{\prime} g\left(f^{\prime 2}-1\right)$. The section of height 49 / 30 must have $x$-coordinate $t(t-1)(t-h)\left(m^{2} t+g^{2}-f^{2}\right)$. Substituting this in to the Weierstrass equation, we obtain a square times an expression quartic in $t$. Completing the square, we obtain two equations. Solving these leads to a rational moduli space with parameters $r$ and $s$.

The universal Weierstrass equation is as follows, where we have scaled $t, x, y$ to avoid denominators:

$$
\begin{aligned}
y^{2}=x^{3}- & \left(4 r^{2} s^{2}+4 r s^{2}-s^{2}+4 r^{2} s+2 r s-r^{2}\right) t^{2}-2\left(2 r^{3} s^{4}\right. \\
& +4 r^{3} s^{3}-4 r^{2} s^{3}+r s^{3}+6 r^{3} s^{2}-5 r^{2} s^{2}+r s^{2}-s^{2}+4 r^{3} s \\
& \left.\left.-7 r^{2} s+2 r s+2 s-3 r^{2}+2 r\right) t-\left(r s^{2}+r s-s-r\right)^{2}\right) x^{2} \\
+ & 8 t((s+1) t-r s-r-s)(r s t-(r-1)(r s-1)) . \\
& \left(\left(4 r^{3} s^{4}+8 r^{3} s^{3}-6 r^{2} s^{3}+r s^{3}+6 r^{3} s^{2}-7 r^{2} s^{2}+3 r s^{2}\right.\right. \\
& \left.\left.-s^{2}+2 r^{3} s-3 r^{2} s+2 r s-r^{2}\right) t+\left(r s^{2}+r s-s-r\right)^{2}\right) x \\
+ & 16 t^{2}((s+1) t-r s-r-s)^{2}(r s t-(r-1)(r s-1))^{2} . \\
& \left(4 r^{2} s^{2}(s+1)(r s+r-1) t+\left(r s^{2}+r s-s-r\right)^{2}\right) .
\end{aligned}
$$

The $A_{2}$ fiber is now at $t=t_{0}=(u-1)(u v-1) /(u v)$.

The $x$-coordinate of a section $P=\left(x_{s}, y_{s}\right)$ of height 49 / 30 is given by

$$
x_{s}=4 t((s+1) t-r s-r-s)(r s t-(r-1)(r s-1))(r s(s+1) t-1) .
$$

For brevity we omit the $y$-coordinate, though it may be found in the auxiliary files.

\subsection{Map to $\mathcal{A}_{2}$ and equation of $\tilde{\mathcal{L}}_{7}$}

We now need to perform "elliptic hopping" to an $E_{8} E_{7}$ fibration. We start with a 2-neighbor step to a fibration with $E_{8}$ and $A_{7}$ fibers. 


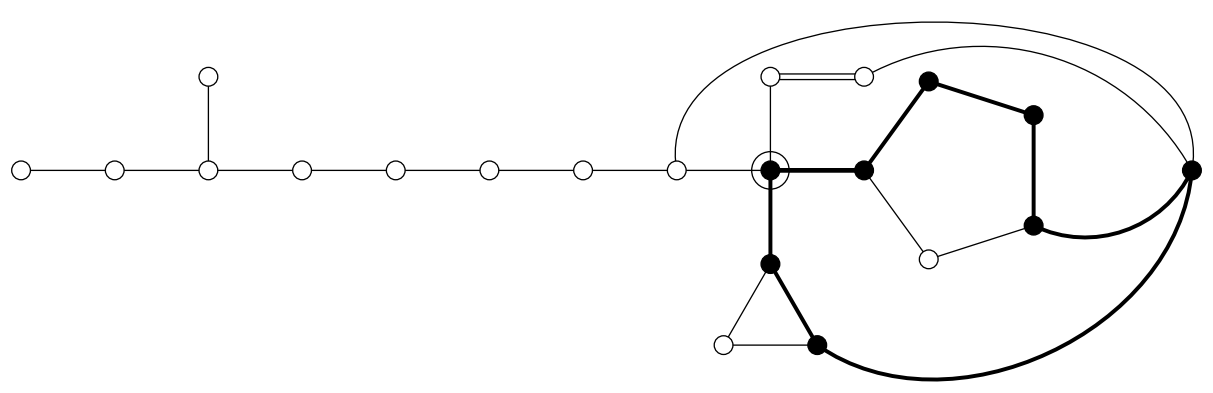

The elliptic parameter is given by

$$
\frac{1}{t\left(t-t_{0}\right)}\left(\frac{y+y_{s}}{x-x_{s}}+\alpha_{0}+\alpha_{1} t\right)
$$

where

$$
\begin{aligned}
& \alpha_{0}=r s^{2}+(r-1) s-r \\
& \alpha_{1}=-\left(2 r^{2} s^{2}+\left(2 r^{2}-r+1\right) s+r^{2}-r\right) /(r-1) .
\end{aligned}
$$

Converting to the Jacobian, it is then an easy matter to take a second 2-neighbor step to an $E_{8} E_{7}$ fibration, as in the second step for discriminant 25 . We omit the details, and also the formulas for the Igusa-Clebsch invariants, which may be found in the auxiliary files.

We then compute the double cover defining the Hilbert modular surface.

Theorem 17 A birational model for the surface $\widetilde{\mathcal{L}}_{7}$ (equivalently, for $Y_{-}(49)$ ) is given by

$$
z^{2}=-16 s^{4} r^{4}+2 s\left(20 s^{2}+17 s-1\right) r^{3}-\left(44 s^{3}+57 s^{2}+18 s-1\right) r^{2}+2 s(15 s+17) r+s^{2} .
$$

It is a singular elliptic K3 surface. The Humbert surface is birational to the $(r, s)$-plane.

This surface has a genus 1 fibration over $\mathbb{P}_{s}^{1}$, with a section, for instance $(z, r)=(s, 0)$. Converting to the Jacobian fibration, we get the Weierstrass equation

$$
\begin{aligned}
y^{2}= & x^{3}+\left(148 s^{3}+45 s^{2}-18 s+1\right) x^{2}+36 s^{4}\left(20 s^{2}+12 s-1\right) x \\
& +64 s^{7}\left(1300 s^{2}+885 s-72\right)
\end{aligned}
$$

of an elliptic K3 surface. It has an $\mathrm{I}_{7}$ fiber at $s=0$, an $\mathrm{I}_{0}^{*}$ fiber at $s=\infty$, a IV fiber at $s=-1$, an $\mathrm{I}_{3}$ fiber at $s=2 / 25$, and $\mathrm{I}_{2}$ fibers at $s=(-5 \pm 2 \sqrt{7}) / 4$. So the contribution to the Picard number from the trivial lattice is 18 . In addition we find the sections

$$
\begin{aligned}
& P=\left(4 s^{3}(4 s-3), 4 s^{3}(s+1)\left(16 s^{2}+40 s-3\right)\right) \\
& Q=\left(-4 s^{2}(5 s-2), 4 s^{2}(s+1)(25 s-2)\right)
\end{aligned}
$$

which are linearly independent. Therefore, the Picard number is 20, i.e. the Hilbert modular surface is a singular K3 surface. The intersection matrix of these sections is

$$
\frac{1}{21}\left(\begin{array}{cc}
13 & 4 \\
4 & 5
\end{array}\right) \text {. }
$$


It has discriminant $1 / 9$. Hence the span of these two sections and the trivial lattice has rank 20 and discriminant $7 \cdot 4 \cdot 3 \cdot 3 \cdot 2^{2} \cdot(1 / 9)=2^{4} \cdot 7$. We check that it is 2 -saturated in the Néron-Severi lattice, and therefore it must be all of NS(Y_(49)).

Once again, we check that the symmetric polynomials of the two $j$-invariants are rational functions of $r$ and $s$ (not displayed here for brevity), and that the difference $j_{1}-j_{2}$ generates the function field of the Hilbert modular surface over that of the Humbert surface. The equation of a tautological family of genus 2 curves may also be found in the computer files.

\subsection{Special loci}

1. The rational curves

$s=-1, z= \pm(r-1)^{2}$

$s=0, z= \pm r$

$r=0, z= \pm s$

$s=\frac{1-r}{r}, z= \pm \frac{r^{3}+5 r^{2}-8 r+1}{r}$

correspond to $I_{10}=0$.

2. The curve $r s=1$ lifts to a rational curve on $Y_{-}$(49). It is part of the product locus.

3. the curve $r=1$ also lifts to a rational curve. It corresponds to the elliptic curves being related by a 13 -isogeny.

4. The curve $r s+r+s=0$ lifts to an elliptic curve on the Hilbert modular surface, isomorphic to $y^{2}+x y+y=x^{3}-x^{2}-x-14$, of conductor 17 and rank 0 . It corresponds to the elliptic curves being related by a 17-isogeny.

5. The curve $s-r\left(s^{2}+s-1\right)=0$ lifts to an elliptic curve on $Y_{-}(19)$, isomorphic to $y^{2}+y=x^{3}+x^{2}-9 x-15$ of conductor 19 and rank 0. It corresponds to the elliptic curves being related by a 19-isogeny.

6. The curve $r s^{3}+2 r s^{2}+s-r+2=0$ lifts to an elliptic curve isomorphic to $y^{2}=x^{3}-x^{2}-4 x+4$, of conductor 24 and rank 0 . It corresponds to the elliptic curves being 24-isogenous.

7. The rational curve $4 r^{2} s^{2}+4 r^{2} s-2 r s-s+r^{2}-r=0$ on the Humbert surface can be parametrized by $(r, s)=\left((t+1) /\left(t^{2}+1\right),\left(t^{2}-1\right) / 4\right)$. It lifts to an elliptic curve on $Y_{-}$(49), isomorphic to $y^{2}+y=x^{3}-7$, which has conductor 27 and rank 0 . It corresponds to the elliptic curves being 27-isogenous.

8. The rational curve $2 r s^{2}+(2 r-1) s+r-1=0$ lifts to an elliptic curve, isomorphic to $y^{2}=x^{3}+x^{2}+4 x+4$ of conductor 20 and rank 0 . It corresponds to the elliptic curves being 20-isogenous.

9. The curve $-16 s^{4} r^{4}+2 s\left(20 s^{2}+17 s-1\right) r^{3}-\left(44 s^{3}+57 s^{2}+18 s-1\right) r^{2}+2 s(15 s+$ 17) $r+s^{2}=0$ has genus 0 and is part of the branch locus. It can be parametrized as

$r=\frac{\left(t^{2}-5\right)\left(t^{2}-2 t-7\right)}{4\left(t^{2}-4 t-1\right)}, \quad s=\frac{4\left(t^{2}-2 t-7\right)}{\left(t^{2}-5\right)^{2}}$

and corresponds to the two elliptic curves being isomorphic.

10. Again, we can obtain several genus 0 non-modular curves from the sections. For instance, the section $P$ gives rise to 


$$
r=\frac{2 s+3}{s(4 s+3)}, \quad z=-\frac{(s+1)\left(16 s^{2}+40 s-3\right)}{(4 s+3)^{2}}
$$

and the section $Q$ to

$r=4 /(5 s), \quad z=(s-2)(25 s-2) /(25 s)$.

The tautological curve along this latter curve is short enough to note here; it is given by

$$
\begin{aligned}
y^{2}= & \left(x^{3}+x^{2}(25 s-2)-8 x(s-4)(25 s-2)-20(s-4)(12 s+1)(25 s-2)\right) \\
& \cdot\left(x^{3}-2 x^{2}(25 s-2) / 5-x(11 s-142)(25 s-2) / 4-5(25 s-2)\left(3 s^{2}\right.\right. \\
& +368 s-148) / 4) .
\end{aligned}
$$

\section{Discriminant 64}

\subsection{Parametrization}

We start with a family of elliptic K3 surfaces with reducible fibers of types $E_{7}, A_{3}$ and $D_{5}$, and a section of height $2=4-3 / 4-5 / 4$. The Weierstrass equation for this family is given by

$$
\begin{gathered}
y^{2}=x^{3}+x^{2} t\left(-4\left(2 s^{5}+6 r s^{4}-s^{4}+4 r^{2} s^{3}+4 r^{2} s^{2}-4 r^{4}\right) t\right. \\
\left.+s^{2}(s+2 r)\left(s^{3}+2 r s^{2}+4 s^{2}+8 r s+8 r^{2}\right)\right) \\
+2 x t^{3}(t-1)\left(-2 s^{3}(s+2 r)^{2}\left(s^{2}+2 s+4 r\right)\left(s^{3}+2 s^{2}+12 r s+8 r^{2}\right) t\right. \\
+2 s^{2}(s+2 r)^{2}\left(3 s^{6}+8 r s^{5}-4 s^{5}+8 r^{2} s^{4}+12 s^{4}\right. \\
\left.\left.+24 r^{2} s^{3}+32 r s^{3}+32 r^{3} s^{2}+32 r^{2} s^{2}+32 r^{3} s+32 r^{4}\right)\right) \\
+t^{4}(t-1)^{2}\left(64 r(s-2) s^{5}(s+r)(s+2 r)^{4}\left(s^{2}+2 s+4 r\right) t\right. \\
\left.+16 s^{4}(s+2 r)^{4}\left(s^{3}-2 s^{2}-4 r s-4 r^{2}\right)^{2}\right) .
\end{gathered}
$$

The $x$-coordinate of a section of height 2 is given by

$$
x_{s}=4\left(s^{2}+2 s+4 r\right)(t-1) t^{2}\left(\left(s^{2}+2 s+4 r\right) t+(s-2 r-2)(s+2 r)\right) .
$$

For brevity, we omit the description of the process of parametrization, which is similar to that for the smaller discriminants described in this paper. A brief outline is given in the auxiliary files, for the interested reader.

\subsection{Map to $\mathcal{A}_{2}$ and equation of $\tilde{\mathcal{L}}_{8}$}

To go to an elliptic fibration with $E_{8}$ and $E_{7}$ fibers, we proceed as follows. First, we take a 3-neighbor step with fiber class as shown below, to go to an elliptic fibration with $D_{8}$ and $E_{7}$ fibers. 


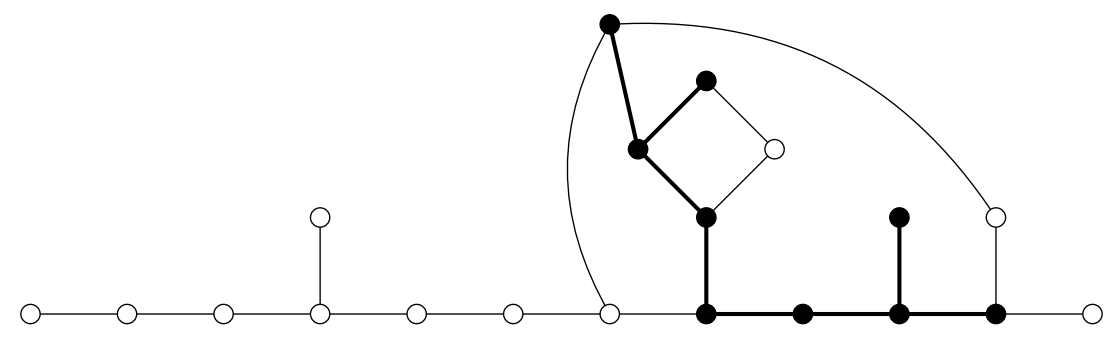

We then take a 2-neighbor step to an $E_{8} E_{7}$ fibration, as in the calculation for discriminant 16 . We omit the details.

The equation for the double cover defining the Hilbert modular surface can now be computed.

Theorem 18 A birational model for the surface $\widetilde{\mathcal{L}}_{8}$ (equivalently, for $Y_{-}(64)$ ) is given by

$$
\begin{aligned}
z^{2}= & -\left(s^{3}+2 s^{2}+12 r s+8 r^{2}\right)(s+2 r+2)\left(27 s^{4}+54 r s^{3}-52 s^{3}-48 r s^{2}\right. \\
& \left.+44 s^{2}+96 r^{2} s+72 r s-16 r^{3}-16 r^{2}\right) .
\end{aligned}
$$

Its minimal model is an honestly elliptic surface $X$ with $\chi\left(\mathcal{O}_{X}\right)=3$ and Picard number 30. The Humbert surface is birational to the $(r, s)$-plane.

The equation above yields an elliptic surface: making the change of variables $r=s t$ and absorbing $s^{4}$ in $z^{2}$ makes the right side quartic in $s$, giving an elliptic fibration over $\mathbb{P}_{t}^{1}$, with an obvious section. Converting to the Jacobian form, after some simple transformations, including the substitution $t=(v-1) / 2$, we obtain the Weierstrass equation

$$
\begin{aligned}
y^{2}= & x^{3}+\left(v^{6}-14 v^{5}+23 v^{4}+108 v^{3}+23 v^{2}-14 v+1\right) x^{2} \\
& -16 v^{4}\left(v^{2}-10 v-7\right)\left(7 v^{2}+10 v-1\right) x
\end{aligned}
$$

which displays an obvious extra symmetry $(x, y, v) \rightarrow\left(x / v^{6}, y / v^{9}, 1 / v\right)$. This is an honestly elliptic surface with $\chi=3$. It has reducible fibers of type $\mathrm{I}_{8}$ at $\infty$ and $0, \mathrm{I}_{2}$ at $1, \mathrm{I}_{4}$ at $-1, \mathrm{I}_{2}$ at the roots of $v^{2}-10 v-7$ and also at the roots of $7 v^{2}+10 v-1$, and $\mathrm{I}_{3}$ at the roots of $v^{2}-10 v+1$. The trivial sublattice has rank 28, leaving room for MordellWeil rank up to 2 . In addition to the 2-torsion section $T=(0,0)$, we find the following sections:

$$
\begin{aligned}
& P=\left(4 v\left(7 v^{2}+10 v-1\right), 4 v(v+1)\left(v^{2}-10 v+1\right)\left(7 v^{2}+10 v-1\right)\right), \\
& Q=\left(4\left(v^{2}-10 v-7\right), 12 \sqrt{-3}(v-1)(v+1)^{2}\left(v^{2}-10 v-7\right)\right) .
\end{aligned}
$$

These sections are orthogonal of heights 1 / 6 and 3 / 2 respectively. Therefore, this elliptic surface has maximal Picard number 30. The discriminant of the lattice generated by $T, P, Q$ and the trivial lattice is $2^{9} \cdot 3^{2}$. We checked that it is 2 - and 3 -saturated. Therefore, it must be the full Picard group, and the Mordell-Weil lattice is generated by these sections. 
The Igusa-Clebsch invariants, the equation of a tautological family of genus 2 curves, and the elementary symmetric polynomials in the $j$-invariants of the associated elliptic curves can be found in the auxiliary files.

\subsection{Special loci}

1. The curve $r=0$ is part of the product locus. It lifts to a genus 0 curve on the Hilbert modular surface, with no real points.

2. The curve $s=0, z=16 r^{2}(r+1)$ has genus 0 and is part of the locus $I_{10}=0$, as is the curve $s=2$, which lifts to a rational curve on $\widetilde{\mathcal{L}}_{8}$. The locus $I_{10}=0$ also contains the curves $r=-s^{2} / 4$ (which lifts to an elliptic curve), $r=-s^{2} / 4-s / 2$ (lifts to a union of two rational curves), and $s^{3}+2 s^{2}+12 r s+8 r^{2}=0$, which has genus 0 and is part of the branch locus. The last curve can be parametrized as $(r, s)=\left(-t\left(1+3 t+t^{2}\right),-2\left(t^{2}+3 t+1\right)\right)$.

3. The curve $s^{3}+2 r s^{2}-2 s^{2}+4 r^{2}=0$ has genus 0 and can be parametrized as $(r, s)=\left(-t\left(t^{2}-2\right) /(2(t+1)),-\left(t^{2}+2\right) /(t+1)\right)$. It lifts to an elliptic curve of conductor 14, and is modular (corresponding to the two elliptic curves being 7-isogenous).

4. The rational curve $s=-2 r-2$ is part of the branch locus, as is the curve

$27 s^{4}+54 r s^{3}-52 s^{3}-48 r s^{2}+44 s^{2}+96 r^{2} s+72 r s-16 r^{3}-16 r^{2}=0$.

The latter can be parametrized as

$$
(r, s)=\left(\frac{\left(t^{2}+26 t+124\right)\left(t^{2}+40 t+408\right)}{216(t+20)}, \frac{(t+16)\left(t^{2}+26 t+124\right)}{54(t+20)}\right)
$$

5. There are several non-modular specializations: for instance, $s \in\{-2,1 / 2,5 / 2\}$ or $r=-1$ all yield elliptic curves with rational points. Of course, we may use the elliptic fibration to produce many elliptic curves, simply by specializing the parameter on the base.

6. The sections of the elliptic fibrations gives us several (usually) non-modular rational curves. For instance, $P$ gives the curve parametrized by

$$
\begin{gathered}
r=-\frac{(v-1)(2 v-1)}{v^{2}-v+1}, \quad s=-\frac{2(2 v-1)}{v^{2}-v+1} \\
z=\frac{16(v-1) v^{2}(v+1)(2 v-1)^{2}\left(v^{2}-10 v+1\right)}{\left(v^{2}-v+1\right)^{4}},
\end{gathered}
$$

while $Q$ gives the curve parametrized by

$$
\begin{aligned}
& r=-\frac{(v-1)\left(7 v^{2}+4 v-7\right)}{(v+1)\left(7 v^{2}-4 v+7\right)}, \quad s=-\frac{2\left(7 v^{2}+4 v-7\right)}{(v+1)\left(7 v^{2}-4 v+7\right)}, \\
& z=\frac{48 \sqrt{-3} v^{3}\left(v^{2}-10 v-7\right)\left(7 v^{2}+4 v-7\right)^{2}\left(7 v^{2}+10 v-1\right)}{(v+1)^{4}\left(7 v^{2}-4 v+7\right)^{4}} .
\end{aligned}
$$

\section{Discriminant 81}

\subsection{Parametrization}

We start with a family of elliptic K3 surfaces with reducible fibers of types $E_{6}, A_{2}$ and $A_{7}$, and a section of height $9 / 8=4-4 / 3-2 / 3-7 / 8$. A Weierstrass equation is given by 


$$
\begin{aligned}
y^{2}= & x^{3}+x^{2}\left(\left(4 r^{2} s^{6}+4 r s^{6}+s^{6}-24 r^{2} s^{5}-24 r s^{5}-6 s^{5}-36 r^{2} s^{4}+108 r s^{4}+15 s^{4}\right.\right. \\
& +240 r^{2} s^{3}+144 r s^{3}-84 s^{3}+252 r^{2} s^{2}-132 r s^{2}-177 s^{2}-216 r^{2} s-504 r s \\
& \left.-198 s+36 r^{2}-108 r-63\right) t^{2}-2\left(2 r^{2} s^{3}+r s^{3}+10 r^{2} s^{2}\right. \\
& \left.\left.+r s^{2}-2 s^{2}-2 r^{2} s-13 r s-4 s+6 r^{2}-5 r-2\right) t+r^{2}\right) \\
& +32 x(s+1)^{2}(r s-r-1) t^{2}\left(4\left(s^{2}+3\right)^{2} t-(s-1)(2 r s+s+2 r-1)\right) \\
& \left(\left(2 r s^{6}+s^{6}-4 r s^{5}-2 s^{5}-16 r^{2} s^{4}+38 r s^{4}+3 s^{4}+64 r^{2} s^{3}+24 r s^{3}-44 s^{3}\right.\right. \\
& \left.+96 r^{2} s^{2}-34 r s^{2}-73 s^{2}-192 r^{2} s-276 r s-114 s+48 r^{2}-6 r-27\right) t^{2} \\
& \left.-2\left(r^{2} s^{3}+r s^{3}+9 r^{2} s^{2}+r s^{2}-2 s^{2}-5 r^{2} s-13 r s-4 s+3 r^{2}-5 r-2\right) t+r^{2}\right) \\
& +256(s+1)^{4}(r s-r-1)^{2} t^{4}\left(4\left(s^{2}+3\right)^{2} t-(s-1)(2 r s+s+2 r-1)\right)^{2} . \\
& \left((s-1)^{2}\left(s^{2}+2 s+8 r+5\right)^{2} t^{2}-2\left(r s^{3}+8 r^{2} s^{2}+r s^{2}-2 s^{2}-8 r^{2} s\right.\right. \\
& \left.-13 r s-4 s-5 r-2) t+r^{2}\right),
\end{aligned}
$$

and the $x$-coordinate of the extra section is

$$
x_{s}=-4(s+1)^{2}(r s-r-1) t\left(4\left(s^{2}+3\right)^{2} t-(s-1)(2 r s+s+2 r-1)\right) /(s-1)^{2} .
$$

\subsection{Map to $\mathcal{A}_{2}$ and equation of $\tilde{\mathcal{L}}_{9}$}

We first go to an elliptic fibration with $E_{8}$ and $A_{7}$ fibers, via the 3-neighbor step corresponding to the figure below.

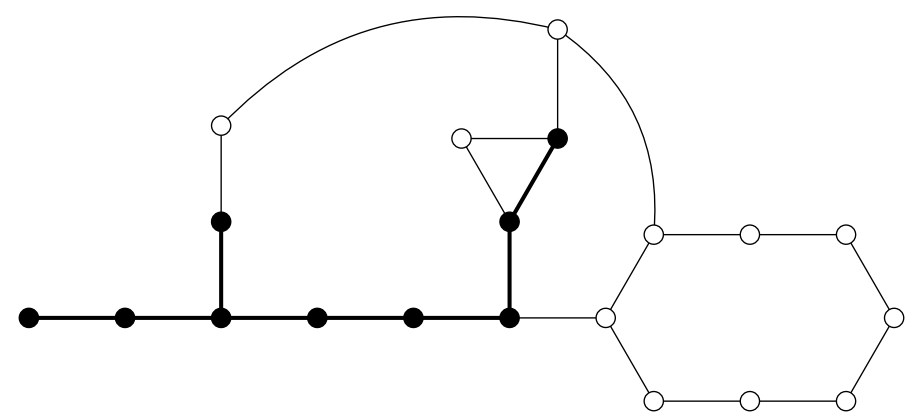

Finally, a 2-neighbor step (as in the second step for discriminant 25) takes us to an $E_{8} E_{7}$ fiber. We can then read out the Igusa-Clebsch invariants, which are in the auxiliary files, along with a tautological family of genus 2 curves and the sum and product of the $j$-invariants of the elliptic curves.

We then compute the double cover defining the Hilbert modular surface.

Theorem 19 A birational model for the surface $\widetilde{\mathcal{L}}_{9}$ (equivalently, for $Y_{-}(81)$ ) is given by

$$
\begin{aligned}
z^{2}= & 16\left(s^{3}-9 s^{2}-9 s+9\right)^{2} r^{4}+32 s\left(s^{5}+5 s^{4}+90 s^{3}+18 s^{2}-171 s-135\right) r^{3} \\
& +8\left(3 s^{6}+40 s^{5}+81 s^{4}-336 s^{3}-627 s^{2}-360 s-81\right) r^{2} \\
& +8(s+1)^{2}\left(s^{4}+5 s^{3}-49 s^{2}-57 s-12\right) r+(s+1)^{4}\left(s^{2}-18 s-15\right) .
\end{aligned}
$$


Its minimal model is an honestly elliptic surface $X$ with $\chi\left(\mathcal{O}_{X}\right)=3$ and Picard number 29. The Humbert surface is birational to the $(r, s)$-plane.

The equation above evidently defines an elliptic surface over $\mathbb{P}_{s}^{1}$ with section. Converting to the Jacobian form, we obtain (after Weierstrass transformations and the change of variable $s=t-1)$

$$
\begin{aligned}
y^{2}= & x^{3}+4\left(44 t^{5}+149 t^{4}-184 t^{3}+280 t^{2}-128 t+16\right) x^{2} \\
& +64 t^{5}\left(125 t^{5}+1403 t^{4}-946 t^{3}+1672 t^{2}+472 t-176\right) x \\
& +256 t^{9}\left(13625 t^{5}-3964 t^{4}+18780 t^{3}+3568 t^{2}+17296 t-4096\right)
\end{aligned}
$$

It is honestly elliptic with $\chi=3$, and reducible fibers of type $\mathrm{I}_{8}$ at $0, \mathrm{I}_{4}^{*}$ at $\infty, \mathrm{I}_{3}$ at the roots of $t^{2}-2 t+4, \mathrm{I}_{2}$ at the roots of $4 t^{3}-12 t^{2}+21 t-4$ and $\mathrm{I}_{3}$ at the roots of $25 t^{3}-66 t^{2}+84 t-16$. These give rise to a trivial lattice of rank 27 , leaving room for Mordell-Weil rank up to 3 . We find the sections

$$
\begin{aligned}
& P=\left(128 t^{3}-256 t^{4}, 16 t^{3}(3 t-4)\left(25 t^{3}-66 t^{2}+84 t-16\right)\right) \\
& Q=\left(16 t^{4}\left(t^{2}-7 t-2\right), 16 t^{4}\left(t^{2}-2 t+4\right)\left(4 t^{3}-12 t^{2}+21 t-4\right)\right)
\end{aligned}
$$

with height matrix

$$
\frac{1}{18}\left(\begin{array}{cc}
18 & -12 \\
-12 & 17
\end{array}\right)
$$

Therefore the Picard number $\rho$ is at least 29. On the other hand, by counting points modulo 7 and 13 and using the method of van Luijk [31] (comparing the square classes of the discriminants of the Picard groups of these reductions) we obtain that the Picard number cannot be 30 . Therefore $\rho=29$ exactly. We checked that the lattice generated by $P$ and $Q$, which has discriminant $2^{4} \cdot 3^{7}$, is 2 - and 3 -saturated. Therefore it is the full Mordell-Weil lattice.

\subsection{Special loci}

1. The curves $r=0, s=-4 r-1$ and $2 r s^{2}-s^{2}-4 r s-2 s+2 r-1=0$ of genus 0 on the Humbert surface are all part of the product locus. The first lifts to a rational curve on $Y_{-}(81)$ and the other two lift to elliptic curves.

2. The curves $s=-1, r s-r-1=0$ and $s^{2}+3=0$ are part of the locus $I_{10}=0$. The first two lift to unions of two rational curves, while the last clearly has no real points.

3. The curve $s=1$ lifts to an elliptic curve isomorphic to $X_{0}(11)$. It corresponds to the two elliptic curves being 11-isogenous.

4. The branch locus has genus 1 , and corresponds to $j_{1}=j_{2}$.

5. The section $P$ gives rise to the following non-modular curve of genus 0 :

$$
\begin{aligned}
& r=-\frac{(s+1)^{2}\left(s^{2}-18 s-27\right)}{(s-3)\left(7 s^{3}+27 s^{2}+45 s+9\right)} \\
& z=\frac{(s-1)(s+1)^{3}\left(25 s^{3}+9 s^{2}+27 s+27\right)\left(s^{4}-18 s^{3}-144 s^{2}-342 s-729\right)}{(s-3)^{2}\left(7 s^{3}+27 s^{2}+45 s+9\right)^{2}} .
\end{aligned}
$$

The section $Q$ gives the non-modular curve 


$$
r=\frac{(s+1)^{2}}{2(s-3) s}, \quad z=-\frac{(s-1)(s+1)^{3}\left(4 s^{3}+9 s+9\right)}{(s-3)^{2} s^{2}} .
$$

The tautological curve over it is given by

$$
\begin{aligned}
& y^{2}=\left(x^{3}+3 x^{2}\left(s^{2}-3 s+6\right)\left(4 s^{3}+9 s+9\right)\right. \\
&- 24 x(s-3) s^{2}\left(s^{2}+3\right)\left(4 s^{2}-3 s+18\right)\left(4 s^{3}+9 s+9\right) \\
&-\left.4(s-3) s\left(4 s^{2}-3 s+18\right)^{2}\left(4 s^{3}+9 s+9\right)^{2}\left(5 s^{3}-9 s^{2}+9 s-9\right)\right) . \\
&\left(x^{3}-6 x^{2}(s-3)\left(s^{2}+3\right)\left(4 s^{2}-3 s+18\right)\right. \\
&-3 x(s+3)\left(4 s^{2}-3 s+18\right)^{2}\left(5 s^{2}+3\right)\left(4 s^{3}+9 s+9\right) / 4 \\
&+\left(4 s^{2}-3 s+18\right)^{3}\left(4 s^{3}+9 s+9\right) . \\
&\left.\quad\left(100 s^{6}-225 s^{5}+945 s^{4}-1062 s^{3}+2106 s^{2}-729 s+81\right) / 4\right) .
\end{aligned}
$$

\section{Discriminant 100}

\subsection{Parametrization}

We start with a family of elliptic K3 surfaces with $D_{6}, A_{6}$ and $A_{3}$ fibers, and a section of height $25 / 12=4-6 / 4-3 / 4-6 / 7$. A Weierstrass equation is given by

$$
\begin{aligned}
& y^{2}=x^{3}+x^{2}(- r^{5}(r+2)^{3}\left(2 s^{2}+2 s-r^{2}\right) t^{3}-r^{2}(r+2)^{2}\left(2 s^{6}+6 s^{5}-5 r^{2} s^{4}\right. \\
&\left.-2 r s^{4}+3 s^{4}-10 r^{2} s^{3}+2 r^{4} s^{2}-2 r^{2} s^{2}+2 r^{4} s-4 r^{3} s-r^{4}\right) t^{2} \\
&+r(r+2) s(s+2)(s-r)(s+r)\left(s^{4}+2 s^{3}-r^{2} s^{2}-2 s^{2}+4 r s+2 r^{2}\right) t \\
&\left.+s^{2}(s+2)^{2}(s-r)^{2}(s+r)^{2}\right) /\left(s^{2}(s+2)^{2}(s-r)^{2}(s+r)^{2}\right) \\
&+2 x r^{3}(r+2)^{3}(s+r+2)(t-1) t^{2}\left(r^{2}(r+2)^{2}\left(s^{2}+s-r^{2}\right) t^{2}\right. \\
&-r(r+2)\left(s^{4}+2 s^{3}-r^{2} s^{2}-s^{2}-r^{2} s+2 r s+r^{2}\right) t \\
&-s(s+2)(s-r)(s+r)) /\left(s(s+2)^{3}(s-r)^{2}(s+r)^{3}\right) \\
&+r^{6}(r+2)^{6}(s+r+2)^{2}(t-1)^{2} t^{4}\left(r^{2} t+2 r t+1\right) /\left((s+2)^{4}(s-r)^{2}(s+r)^{4}\right),
\end{aligned}
$$

and the extra section having $x$-coordinate

$$
x_{s}=s^{3}(s+2)(s-r)^{2}(s+r)(s+r+2) t\left(t-r^{2}-2 r\right) / r^{2} .
$$

\subsection{Map to $\mathcal{A}_{2}$ and equation of $\tilde{\mathcal{L}}_{10}$}

We first go to an elliptic fibration with $E_{7}, A_{7}$ and $A_{1}$ fibers by a 2-neighbor step.

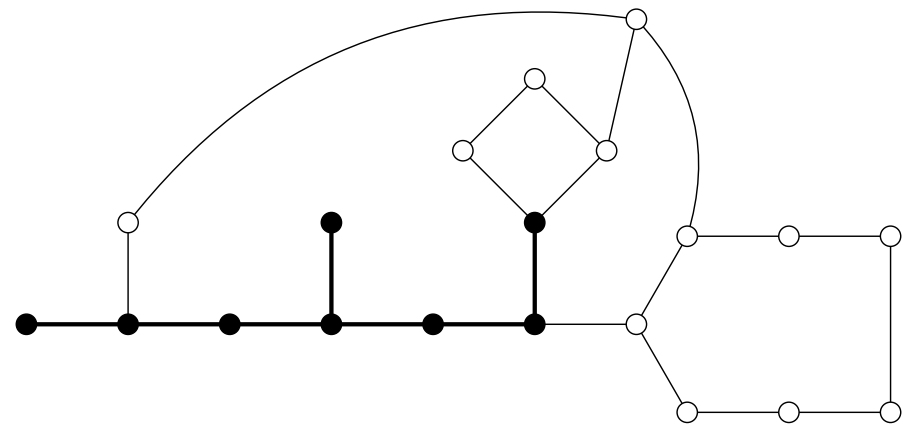

Next, we go to a $D_{6} D_{5} A_{3}$ fibration, via the fiber class shown below. 


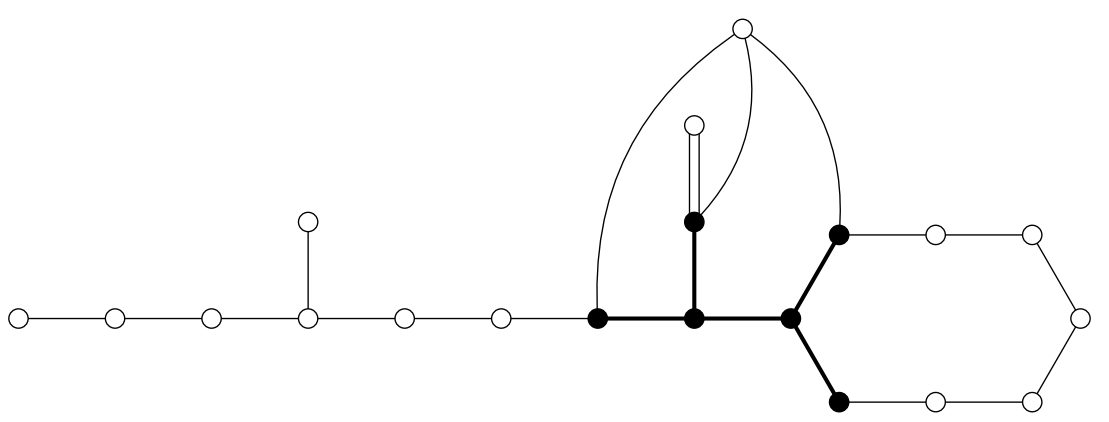

The new elliptic fibration has Mordell-Weil group of rank 2, generated by two sections $P$ and $Q$ with height matrix

$$
\frac{1}{4}\left(\begin{array}{cc}
2 & 1 \\
1 & 13
\end{array}\right)
$$

with the entries arising as

$$
\begin{aligned}
1 / 2 & =4-3 / 4-5 / 4-6 / 4, \\
13 / 4 & =4-3 / 4 \\
1 / 4 & =2-1-3 / 4
\end{aligned}
$$

A 2-neighbor step takes us to a fibration with $D_{8} D_{6} A_{1}$ fibers, a 2-torsion section $T$ (and a section of height 25 / 2).

The section $Q$ intersects the fiber class in 3, whereas the remaining component of the $D_{5}$ fiber intersects it in 2 . Since these are coprime, the new fibration has a section. Note also that the section $2 P$ of height $2=4-1-2 \cdot 2 / 4$ doesn't intersect the fiber class, giving rise to an extra $A_{1}$ fiber.

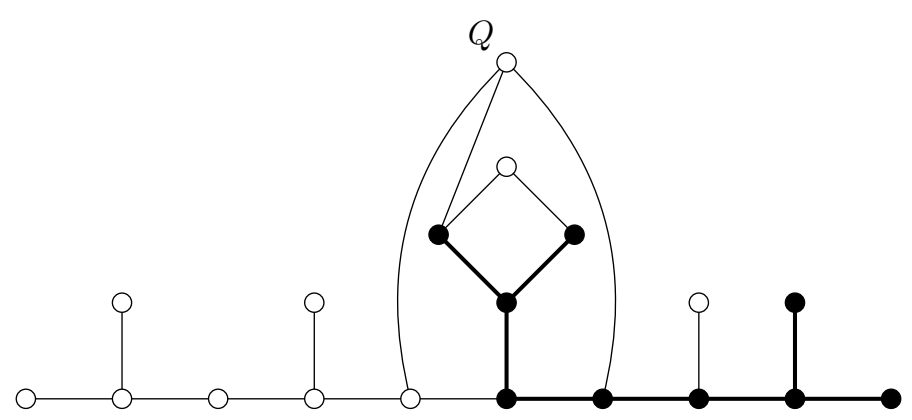

Finally, a 2-neighbor step leads us to the desired $E_{8} E_{7}$ fibration. The fiber $F$ with an extended $E_{8}$ Dynkin diagram is shown below, and the 2-torsion section $T$ combines with some of the $D_{6}$ components and the non-identity component of the $A_{1}$ fiber to give an $E_{7}$ diagram disjoint from $F$. Also, the section $P$ of height $25 / 2$, or its negative, has odd intersection number with $F$, while the remaining component of the $D_{8}$ fiber has intersection 2 with $F$. So the new fibration has a section. We can read out the IgusaClebsch invariants, which are in the auxiliary files. 


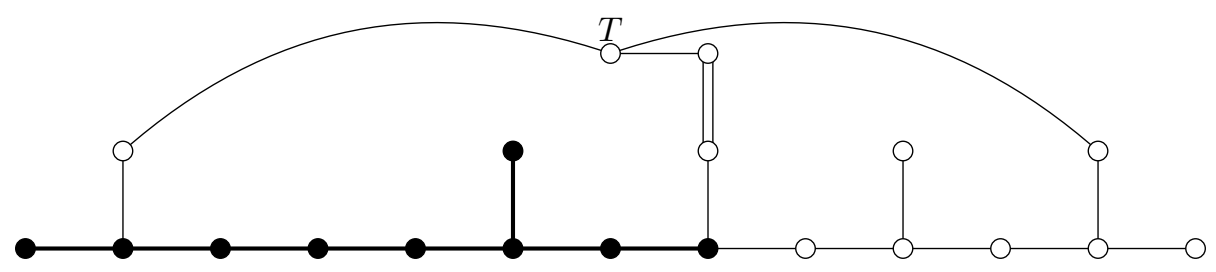

We compute the double cover defining the Hilbert modular surface.

Theorem 20 A birational model for the surface $\widetilde{\mathcal{L}}_{10}$ (equivalently, for $Y_{-}(100)$ ) is given by

$$
\begin{gathered}
z^{2}=-\left(2 s^{2}+2 s-r^{2}\right)\left(s^{6}-2 r s^{5}-r^{2} s^{4}+2 r s^{4}+2 s^{4}+4 r^{3} s^{3}+2 r^{2} s^{3}+12 r s^{3}+14 s^{3}\right. \\
\left.-2 r^{4} s^{2}-14 r^{3} s^{2}-29 r^{2} s^{2}-20 r s^{2}+10 r^{4} s+10 r^{3} s-2 r^{2} s+r^{4}\right) .
\end{gathered}
$$

Its minimal model is an honestly elliptic surface $X$ with $\chi\left(\mathcal{O}_{X}\right)=3$ and Picard number 28 or 29. The Humbert surface is birational to the $(r, s)$-plane.

Making the substitution $r=s t$ and absorbing $s^{4}$ in to $z^{2}$ makes the right side quartic in $s$, showing that $\mathcal{L}_{10}$ is an elliptic surface with section over $\mathbb{P}_{t}^{1}$. Converting to the Jacobian form, after some Weierstrass transformations and substitution $t=u+1$, we obtain

$$
\begin{aligned}
y^{2}= & x^{3}+\left(u^{6}+16 u^{5}+74 u^{4}+92 u^{3}+21 u^{2}+20 u+4\right) x^{2} \\
& +8 u^{2}\left(10 u^{5}+119 u^{4}+324 u^{3}+291 u^{2}+68 u+4\right) x \\
& -16 u^{4}\left(8 u^{4}+84 u^{3}+139 u^{2}+12 u-4\right) .
\end{aligned}
$$

It is an honestly elliptic surface with $\chi=3$, and reducible fibers of type $\mathrm{I}_{10}$ at $\infty, \mathrm{I}_{5}$ at 0 , IV at $-2, \mathrm{I}_{2}$ at the roots of $u^{2}+3 u+1$ and also at the roots of $u^{2}+8 u-4$, and $\mathrm{I}_{3}$ at the roots of $3 u^{3}+26 u^{2}+14 u+2$. The trivial lattice has rank 27, leaving room for MordellWeil rank up to 3 . We find the section

$$
P=\left(-4 u^{2}\left(3 u^{2}+26 u+5\right), 4 u^{2}\left(u^{2}+8 u-4\right)\left(3 u^{3}+26 u^{2}+14 u+2\right)\right)
$$

of height $1 / 5$. Therefore, the Picard number $\rho$ is at least 28 . On the other hand, counting points modulo 7 and 17 shows that $\rho<30$. Therefore the Picard number is either 28 or 29; we have not been able to determine it exactly.

The equation of a tautological family of genus 2 curves and the elementary symmetric polynomials in the $j$-invariants of the two elliptic curves are omitted here, but can be found in the computer files.

\subsection{Special loci}

1. The rational curves $s^{2}+2 s-r^{2}=0$ (parametrization $r=-t(t+2) /(2(t+1))$, $\left.s=t^{2} /(2(t+1))\right)$ and $s^{2}+r s+2 s-r^{2}=0$ (parametrization $r=2(t-3)(3 t-4) /$ $\left.\left(5\left(t^{2}-t-1\right)\right), s=2(t-3)^{2} /\left(5\left(t^{2}-t-1\right)\right)\right)$ are part of the product locus, and lift to elliptic curves on $\widetilde{\mathcal{L}}_{10}$. The rational curve $2 s^{2}+2 s-r^{2}=0$ (parametrized by $\left.r=-2 t /\left(t^{2}-2\right), s=2 /\left(t^{2}-2\right)\right)$ is also part of the product locus, as well as the branch locus. 
2. The curves $s=-2, s=-r, s=0, s=r$ and $s=-r-2$ are part of the locus $I_{10}=0$. The first two lift to rational curves, while the others lift to unions of two rational curves.

3. The curve $r=0$ lifts to an elliptic curve isomorphic to $X_{0}(11)$. It corresponds to the two elliptic curves being 11-isogenous.

4. The curve $r=-2$ lifts to an elliptic curve isomorphic to $X_{0}(19)$. It corresponds to the elliptic curves being 19-isogenous.

5. The curve $s=-1$ also lifts to an elliptic curve (with conductor 36 and CM), and is modular: it corresponds to the elliptic curves being 9-isogenous.

6. The curve $r=-1$ lifts to a rational curve; the two elliptic curves are again 9-isogenous along that locus.

7. The branch locus is an elliptic curve, and corresponds to $j_{1}=j_{2}$.

8. The section above gives rise to the non-modular genus 2 curve parametrized by

$$
\begin{aligned}
& r=2(u+1)(3 u+1) /\left(u\left(3 u^{2}+8 u+2\right)\right), \\
& s=2(3 u+1) /\left(u\left(3 u^{2}+8 u+2\right)\right), \\
& z=8(3 u+1)^{2}\left(u^{2}+3 u+1\right)\left(3 u^{3}+26 u^{2}+14 u+2\right) /\left(u^{3}\left(3 u^{2}+8 u+2\right)^{4}\right) .
\end{aligned}
$$

\section{Discriminant 121}

\subsection{Parametrization}

Finally, we describe moduli space of elliptic subfields of degree 11 via the Hilbert modular surface of discriminant 121 . To compute the surface, we work with the moduli space of elliptic K3 surfaces with $A_{6}, A_{5}$ and $A_{3}$ fibers, and two sections with intersection matrix

$$
\frac{1}{84}\left(\begin{array}{cc}
59 & -46 \\
-46 & 61
\end{array}\right)
$$

with the entries arising as

$$
\begin{aligned}
59 / 84 & =4-3 / 4-5 / 6-12 / 7 \\
61 / 42 & =4-5 / 6-12 / 7 \\
-23 / 42 & =2-5 / 6-12 / 7
\end{aligned}
$$

We will be very brief in our description of all the steps, since the formulas are all fairly complicated and explained in greater length in the auxiliary files.

The moduli space of these K3 surfaces is again rational, and with chosen parameters $r$ and $s$, the universal Weierstrass equation is as follows.

$$
y^{2}=x^{3}+a(t) x^{2}+b(t) x+c(t),
$$

where 


$$
\begin{aligned}
& a(t)=(s-r)^{2} t^{4}-16 t^{3} r\left(r s^{4}+3 s^{4}-2 r^{2} s^{3}-5 r s^{3}-4 s^{3}+r^{3} s^{2}+4 r^{2} s^{2}+5 r s^{2}\right. \\
& \left.+2 s^{2}-2 r^{3} s-4 r^{2} s-4 r s-s+r^{3}+2 r^{2}+r\right) / s \\
& +64 r(s-1)^{2} t^{2}\left(r^{3} s^{4}+6 r^{2} s^{4}+7 r s^{4}+4 s^{4}-2 r^{4} s^{3}-8 r^{3} s^{3}-12 r^{2} s^{3}-10 r s^{3}\right. \\
& -6 s^{3}+r^{5} s^{2}+4 r^{4} s^{2}+7 r^{3} s^{2}+6 r^{2} s^{2}+5 r s^{2}+2 s^{2}-2 r^{5} s \\
& \left.-6 r^{4} s-10 r^{3} s-10 r^{2} s-4 r s+r^{5}+4 r^{4}+6 r^{3}+4 r^{2}+r\right) / s^{2} \\
& -1024 \operatorname{tr}(s-1)^{2}(s-r-1)\left(r^{3} s^{4}+5 r^{2} s^{4}+4 r s^{4}-2 s^{4}-r^{4} s^{3}-5 r^{3} s^{3}-8 r^{2} s^{3}\right. \\
& +r s^{3}+4 s^{3}+r^{4} s^{2}-8 r^{2} s^{2}-10 r s^{2}-2 s^{2}+r^{4} s \\
& \left.+7 r^{3} s+12 r^{2} s+6 r s-r^{4}-3 r^{3}-3 r^{2}-r\right) / s^{2} \\
& +4096 r^{2}(s-1)^{4}(s-r-1)^{2}(r s+2 s-r-1)^{2} / s^{2}, \\
& b(t)=512 t^{2} r(s-1)^{2}(s-r-1)\left(s^{2}-r^{2} s-2 r s-s+r^{2}+r\right)\left(t-8\left(r^{2} s\right.\right. \\
& \left.\left.+r s-s-r^{2}+1\right)\right) \cdot\left(s(s-r)^{2} t^{3}-8 t^{2} r\left(2 r s^{4}+5 s^{4}-3 r^{2} s^{3}\right.\right. \\
& -7 r s^{3}-5 s^{3}+r^{3} s^{2}+4 r^{2} s^{2}+3 r s^{2}+s^{2}-2 r^{3} s-3 r^{2} s \\
& \left.-3 r s-s+r^{3}+2 r^{2}+r\right)+64 r(s-1)^{2} t\left(r^{3} s^{3}+5 r^{2} s^{3}+5 r s^{3}-r^{4} s^{2}\right. \\
& -4 r^{3} s^{2}-5 r^{2} s^{2}+r s^{2}+s^{2} \\
& \left.-3 r^{3} s-10 r^{2} s-8 r s-s+r^{4}+4 r^{3}+5 r^{2}+2 r\right) \\
& \left.-512 r^{2}(s-1)^{3}(s-r-1)(r s+2 s-r-1)^{2}\right) / s^{2}, \\
& c(t)=65536 t^{4} r^{2}(s-1)^{4}(s-r-1)^{2}\left(s^{2}-r^{2} s-2 r s-s+r^{2}+r\right)^{2} \\
& \times\left(t-8\left(r^{2} s+r s-s-r^{2}+1\right)\right)^{2} . \\
& \times\left((s-r)^{2} t^{2}-16 r\left(r s^{3}+2 s^{3}-r^{2} s^{2}-2 r s^{2}-s^{2}-2 r s-s+r^{2}+r\right) t\right. \\
& \left.+64 r^{2}(s-1)^{2}(r s+2 s-r-1)^{2}\right) / s^{2} .
\end{aligned}
$$

The $x$-coordinates of the two sections are given by

$$
\begin{aligned}
x_{1}= & 256 \operatorname{tr}(s-1)^{2}(s-r-1)\left(8 r^{2} s+8 r s-8 s-8 r^{2}-t+8\right) \\
\times\left(s^{2}-r^{2} s-2 r s-s+r^{2}+r\right) / s & \\
x_{2}=- & 256 \operatorname{tr}(s-1)^{2}(s-r-1)\left(s\left(s^{2}-r^{2} s-2 r s-s+r^{2}+r\right) t\right. \\
& \left.-8 r(r s+2 s-r-1)^{2}\left(s^{2}-r-1\right)\right) / s^{2} .
\end{aligned}
$$

12.2 Map to $\mathcal{A}_{2}$ and equation of $\tilde{\mathcal{L}}_{11}$

We next quickly illustrate the neighbor steps required to go to an $E_{8} E_{7}$ fibration. 


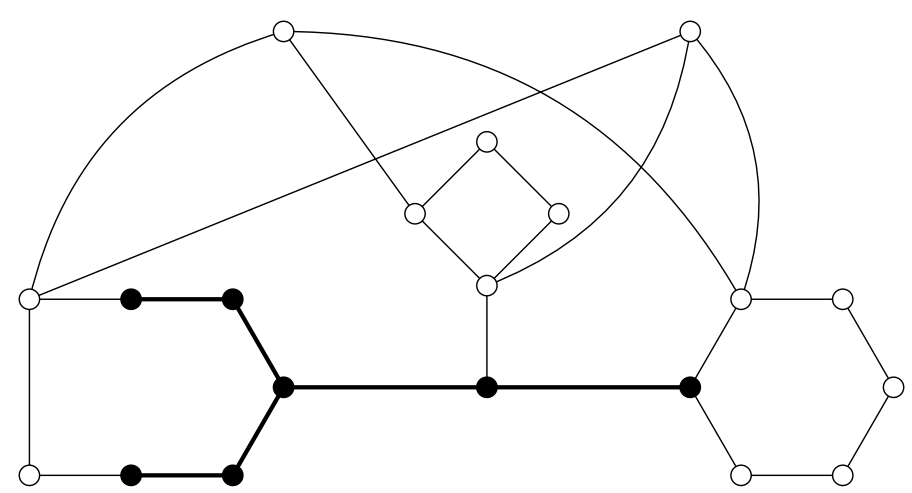

First, we take a 2-neighbor step to an elliptic fibration with $E_{6}, A_{4}, A_{3}$ and $A_{1}$ fibers, and two sections with intersection matrix

$$
\frac{1}{60}\left(\begin{array}{cc}
37 & 25 \\
25 & 115
\end{array}\right)
$$

with the entries being realized as

$$
\begin{aligned}
37 / 60 & =4-4 / 3-1 / 2-4 / 5-3 / 4 \\
23 / 12 & =4-4 / 3-3 / 4 \\
5 / 12 & =2-4 / 3-1 / 4 .
\end{aligned}
$$

In the figure below we only display the section of height $23 / 12$, in order to not clutter the picture.

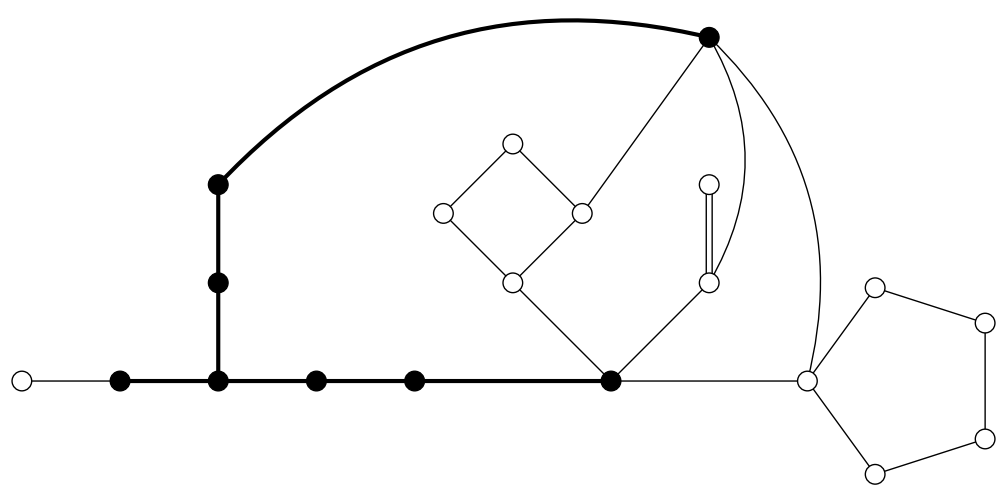

Next, we go by a 2-neighbor step to an $E_{7} A_{4} A_{2} A_{1}$ fibration with two sections having intersection matrix

$$
\frac{1}{30}\left(\begin{array}{rr}
16 & -5 \\
-5 & 115
\end{array}\right)
$$

with the entries being realized as

$$
\begin{aligned}
8 / 15 & =4-3 / 2-4 / 5-2 / 3-1 / 2 \\
23 / 6 & =6-3 / 2-2 / 3 \\
-1 / 6 & =2+1-1-3 / 2-2 / 3 .
\end{aligned}
$$


We only show the section of height 8 / 15 below.

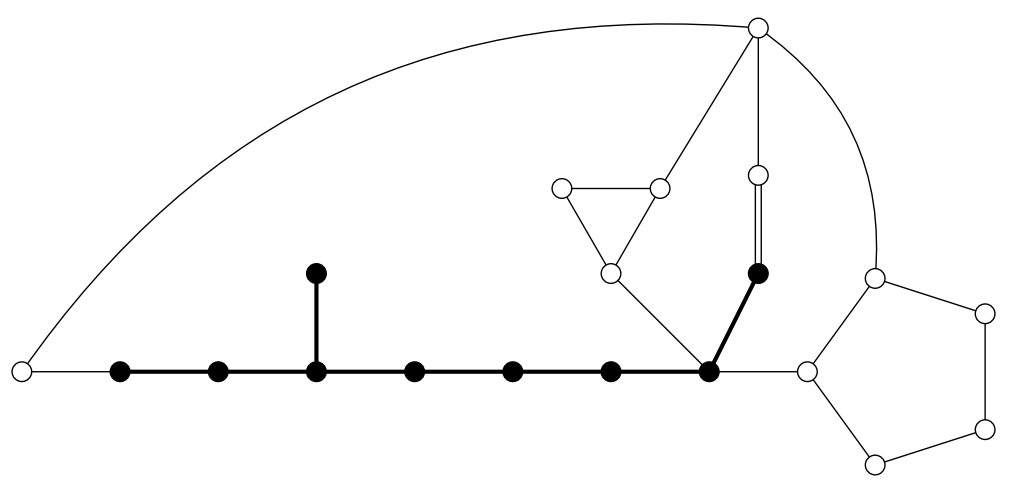

We next take a 2-neighbor step to an $E_{8} A_{7}$ fibration, with a section of height $121 / 8=4+2 \cdot 6-7 / 8$. Finally, we take a 2 -neighbor step to go to an $E_{8} E_{7}$ fibration, as in the second step for discriminant 25. The formulas for the Igusa-Clebsch invariants can now be read off; they may be found in the auxiliary files.

Finally, we compute the double cover defining the Hilbert modular surface.

Theorem 21 A birational model for the surface $\widetilde{\mathcal{L}}_{11}$ (equivalently, for $Y_{-}(121)$ ) is given by

$$
\begin{aligned}
z^{2}=( & -1)^{4} r^{6}-2(s-1)^{3}\left(s^{2}+4 s+2\right) r^{5}+(s-1)^{2}\left(s^{4}-4 s^{3}-40 s^{2}-10 s+6\right) r^{4} \\
& +2(s-1)\left(6 s^{5}-3 s^{4}-25 s^{3}-22 s^{2}+22 s-2\right) r^{3} \\
& +\left(22 s^{6}-8 s^{5}-10 s^{4}-108 s^{3}+113 s^{2}-26 s+1\right) r^{2} \\
& -2(s-1) s^{2}(s+1)(2 s-13)(2 s-1) r-27(s-1)^{2} s^{4}
\end{aligned}
$$

It is a surface of general type. The Humbert surface is birational to the $(r, s)$-plane.

The expressions for the sum and product of the $j$-invariants of the two associated elliptic curves may be found in the auxiliary computer files, which also have the equation of a tautological family of genus 2 curves.

\subsection{Special loci}

Once again we describe some special curves of low genus on the modular surface.

1. The rational curve $s=0$ and the genus 1 curve $s^{2}-r^{2} s-2 r s-s+r^{2}+r=0$ are contained in the product locus. Each lifts to a union of two curves on the Humbert surface (of genera 0 and 1 respectively).

2. The curves

$$
\begin{aligned}
& r=0 \\
& s=1 \\
& s=r+1 \\
& r s+s-r=0 \\
& r^{2} s+r s-s-r^{2}+1=0 \\
& s^{2}-r^{2} s-r s-s+r^{2}=0 \\
& r s^{2}+2 s^{2}-r^{2} s-3 r s-2 s+r^{2}+r=0
\end{aligned}
$$


all correspond to $I_{10}=0$. The first five are of genus 0 and the remaining two have genus 1 . The first two lift to unions of two genus 0 curves with no rational points, the third to a union of two $\mathbb{P}^{1}$ s, the fourth to an irreducible $\mathbb{P}^{1}$, the fifth to an elliptic curve, and the last two to curves of genus 2 and 3 respectively.

3. The rational curve $r s+2 s-r-1=0$ lifts to an elliptic curve isomorphic to $X_{0}(21)$, and corresponds to the elliptic curves being 21-isogenous.

4. the rational curve $r=-1$ lifts to an elliptic curve isomorphic to $X_{0}(17)$, and corresponds to the elliptic curves being 17 -isogenous.

5. The rational curve $s^{2}+r s-r=0$ lifts to an elliptic curve isomorphic to $X_{0}(32)$, and corresponds to the the elliptic curves being 32 -isogenous.

\section{Author details}

${ }^{1}$ Department of Mathematics, Massachusetts Institute of Technology, Cambridge, MA 02139, USA. ${ }^{2}$ Present Address: Department of Mathematics, Stony Brook University, Stony Brook, NY 11794, USA.

Received: 9 June 2015 Accepted: 30 September 2015

Ter.

References

1. An, S.Y., Kim, S.Y., Marshall, D.C., Marshall, S.H., McCallum, W.G., Perlis, A.: Jacobians of genus one curves. J. Number Theory $\mathbf{9 0}$ (2), 304-315 (2001)

2. Bruin, N., Doerksen, K.: The arithmetic of genus two curves with (4,4)-split Jacobians. Can. J. Math. 63, 992-1021 (2011)

3. Bruin, N., Flynn, E.V., Testa, D.: Descent via (3, 3)-isogeny on Jacobians of genus 2 curves. Acta Arith. 165(3), 201-223 (2014)

4. Carlton, D.: Moduli for pairs of elliptic curves with isomorphic N-torsion. Manuscr. Math. 105(2), 201-234 (2001)

5. Cassels, J.W.S., Flynn, E.V.: Prolegomena to a middlebrow arithmetic of curves of genus 2, London Mathematical Society Lecture Note Series, 230. Cambridge University Press, Cambridge (1996)

6. Dolgachev I.: Endomorphisms of complex abelian varieties. http://www.math.Isa.umich.edu/ idolga/MilanLect.pdf

7. Elkies, N.D., Kumar, A.: K3 surfaces and equations for Hilbert modular surfaces. Algebra Number Theory $\mathbf{8}(10)$, 2297-2411 (2014)

8. Frey, G.: On elliptic curves with isomorphic torsion structures and corresponding curves of genus 2, Elliptic curves, modular forms \& Fermat's last theorem (Hong Kong, 1993). Ser. Number Theory I, pp. 79-98. Int. Press, Cambridge (1995)

9. Frey, G., Kani, E.: Curves of genus 2 covering elliptic curves and an arithmetical application, Arithmetic algebraic geometry (Texel, 1989) Progr. Math. 89, pp. 153-176. Birkhäuser Boston, Boston (1991)

10. Gross, B.H., Zagier, D.B.: Heegner points and derivatives of L-series. Invent. Math. 84(2), 225-320 (1986)

11. Harder, A.J.: Moduli spaces of K3 surfaces with large Picard number, Master's thesis. Queen's University (2011)

12. Hausmann, W.: The fixed points of the symmetric Hilbert modular group of a real quadratic field with arbitrary discriminant. Math. Ann. 260(1), 31-50 (1982)

13. Hermann, C.F.: Modulfächen quadratischer Diskriminante. Manuscr. Math. 72(1), 95-110 (1991)

14. Igusa, J.: Arithmetic variety of moduli for genus two, Ann. Math. (2) 72, 612-649 (1960)

15. Krazer, A.: Lehrbuch der Thetafunctionen. Chelsea, New York (1970)

16. Kuhn, R.M.: Curves of genus 2 with split Jacobian. Trans. Am. Math. Soc. 307(1), 41-49 (1988)

17. Kumar, A.: K3 surfaces associated with curves of genus two, Int. Math. Res. Not. IMRN , no. 6, Art. ID rnm165, pp. 26 (2008)

18. Kumar, A.: Elliptic fibrations on a generic Jacobian Kummer surface. J. Algebraic Geom. 23, 599-667 (2014)

19. Kumar, A., Mukamel, R.: Algebraic models and arithmetic geometry of Teichmüller curves in genus two, preprint. arXiv:1406.7057

20. Kumar, A., Mukamel, R.: Real multiplication through explicit correspondences (in preparation)

21. Kani, E., Schanz, W.: Modular diagonal quotient surfaces. Math. Z. 227(2), 337-366 (1998)

22. Mazur, B.: Modular curves and the Eisenstein ideal. Publications Mathématiques de l'Institut des Hautes Études Scientifiques 47(1), 33-186 (1977)

23. Magaard, K., Shaska, T., Völklein, H.: Genus 2 curves that admit a degree 5 map to an elliptic curve. Forum Math. $\mathbf{2 1}(3), 547-566$ (2009)

24. McMullen, C.T.: Foliations of Hilbert modular surfaces. Am. J. Math. 129, 183-215 (2007)

25. Murabayashi, N.: The moduli space of curves of genus two covering elliptic curves. Manuscripta Math. 84(2), 125-133 (1994)

26. Shaska, T:: Genus 2 fields with degree 3 elliptic subfields. Forum Math. 16(2), 263-280 (2004)

27. Shaska, T., Völklein H.: Elliptic subfields and automorphisms of genus 2 function fields, Algebra, Arithmetic and Geometry with Applications. Papers from Shreeram S. Abhyankars 70th Birthday Conference, pp. 687-07, Springer (2004) 
28. Shaska, T., Wijesiri, G.S., Wolf, S., Woodland, L.: Degree 4 coverings of elliptic curves by genus 2 curves. Albanian J. Math. 2(4), 307-318 (2008)

29. Tate, J.: Algorithm for determining the type of a singular fiber in an elliptic pencil, Modular functions of one variable, IV (Proc. Internat. Summer School, Univ. Antwerp, Antwerp, 1972), vol. 476, pp. 33-52. Lecture Notes in Math., Springer, Berlin (1975)

30. van der Geer, G.: Hilbert Modular Surfaces, Ergebnisse der Mathematik und ihrer Grenzgebiete (3), 16. SpringerVerlag, Berlin (1988)

31. van Luijk, R.: K3 surfaces with Picard number one and infinitely many rational points. Algebra Number Theory $\mathbf{1}(1)$, $1-15$ (2007)

32. van Wamelen, P.: Proving that a genus 2 curve has complex multiplication. Math. Comp. 68(228), 1663-67 (1999)

33. van Wamelen, P.: Computing with the analytic Jacobian of a genus 2 curve. In: Discovering mathematics with Magma, pp. 117-135, Springer (2006)

34. Wiles, A.: Modular elliptic curves and Fermat's last theorem, Ann. Math. (2). 141(3):443-551 (1995)

Submit your manuscript to a SpringerOpen ${ }^{\circ}$ journal and benefit from:

- Convenient online submission

- Rigorous peer review

- Immediate publication on acceptance

- Open access: articles freely available online

- High visibility within the field

- Retaining the copyright to your article

Submit your next manuscript at $\boldsymbol{s p r i n g e r o p e n . c o m ~}$ 